\title{
Power Efficient Scheduling and Hybrid Precoding for Time Modulated Arrays
}

This paper was downloaded from TechRxiv (https://www.techrxiv.org).

LICENSE

CC BY 4.0

SUBMISSION DATE / POSTED DATE

$16-12-2019$ / 31-12-2019

CITATION

González-Coma, José P.; Castedo, Luis (2019): Power Efficient Scheduling and Hybrid Precoding for Time Modulated Arrays. TechRxiv. Preprint. https://doi.org/10.36227/techrxiv.11372100.v2

$\mathrm{DOI}$

10.36227/techrxiv.11372100.v2 


\title{
Power Efficient Scheduling and Hybrid Precoding for Time Modulated Arrays
}

\author{
José P. González-Coma, Member, IEEE, and Luis Castedo, Senior Member, IEEE
}

\begin{abstract}
We consider power efficient scheduling and precoding solutions for multiantenna hybrid digital-analog transmission systems that use Time-Modulated Arrays (TMAs) in the analog domain. TMAs perform beamforming with switches instead of conventional Phase Shifters (PSs). The extremely low insertion losses of switches, together with their reduced power consumption and cost make TMAs attractive in emerging technologies like massive Multiple-Input Multiple-Output (MIMO) and millimeter wave (mmWave) systems. We propose a novel analog processing network based on TMAs and provide an angular scheduling algorithm that overcomes the limitations of conventional approaches. Next, we pose a convex optimization problem to determine the analog precoder. This formulation allows us to account for the Sideband Radiation (SR) effect inherent to TMAs, and achieve remarkable power efficiencies with a very low impact on performance. Computer experiments results show that the proposed design, while presenting a significantly better power efficiency, achieves a throughput similar to that obtained with other strategies based on angular selection for conventional architectures.
\end{abstract}

\section{INTRODUCTION}

Massive Multiple-Input Multiple-Output (MIMO) relies on the use of a large number of antennas to take advantage of the wireless channel spatial diversity and achive high beamforming gains [1]. Massive MIMO is particularly feasible at millimeter wave (mmWave) where the small wavelengths handled allow the integration of large antenna arrays into apertures of reasonable size [2]. Nevertheless, precoding for these large antenna arrays is costly and power hungry, specially when exploiting the huge signal bandwidths at mmWave. To alleviate these issues, it is common to consider hybrid digital-analog precoding (see Fig. 1) as a tradeoff between cost and performance [3]. In this scheme the number of Radio Frequency (RF) chains, $N_{\mathrm{RF}}$, is much smaller that the number of deployed antennas, $M$.

Time-Modulated Array (TMA) is an unconventional way to perform analog precoding using switches operating periodically instead of Phase Shifters (PSs) [4]. From a circuit perspective, RF switches exhibit significant advantages with respect to PSs like lower Insertion Lossess (ILs), less power consumption, reduced implementation costs and absence of quantization errors [5]. TMAs, on the other hand, suffer from Sideband Radiation (SR), i.e., the radiation of signal power over the TMA harmonic frequencies. If only the TMA central frequency (referred to as the fundamental mode) is exploited, $\mathrm{SR}$ is an undesired phenomenon. Conventional TMAs with

José P. González-Coma and Luis Castedo are with the University of A Coruña, CITIC, Spain. e-mail: \{jose.gcoma, luis\}@udc.es

This work has been funded by AEI of Spain (TEC2016-75067-C4-1-R, RED2018-102668-T), and ERDF funds (AEI/FEDER, EU).

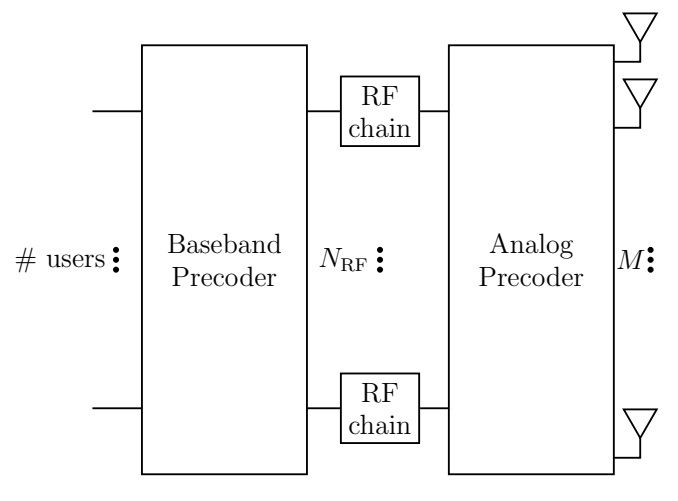

Fig. 1: Hybrid digital-analog precoding.

Single-Pole-Single-Throw (SPST) switches also suffer from hardware inefficiencies due to the absorptive losses during the OFF-state of the switches [4].

To improve the TMA hardware efficiency, solutions based on Single-Pole-Double-Throw (SPDT) switches have been proposed, although they yield to stringent restrictions in the analog precoding matrix [6]. A more sophisticated solution was recently proposed in [7] using Reconfigurable Power Dividers/Combiners. This approach, however, is more complex as it requires additional hardware, and the flexibility to design the analog precoder is also limited. Indeed, the synthesis of conventional array weighting patterns has only been shown for symmetric weighting vectors. This limitation imposes severe constraints when designing analog precoding matrices, especially in multiuser scenarios.

Recent work on hybrid digital-analog precoding shows the suitability of TMAs to develop low-cost analog processing networks [4]. Therein, the authors pose an architecture employing SPST switches and simple 1-bit PSs to obtain signed realvalued analog precoding matrices. However, this switching network results in hardware inefficiencies that are difficult to compensate. In addition, the hybrid precoding designs presented in [4] only apply in single user scenarios. Downlink setups have been considered in [8] and [9]. While [8] considers fixed user directions matching the beams of the different harmonics, [9] assumes the unlikely scenario of isotropically distributed users. Moreover, both approaches employ rigid weighting patterns to design the precoding matrix, leading to inefficient power allocations.

Unlike the solutions in [8], [9], we propose in this work an alternative TMA analog network that provides total flexibility in the design of the analog precoder. This freedom to design the precoding matrix was achieved in [4], but the approach 
now proposed avoids the power losses caused by the SPST switches during the OFF-state. Our novel scheme is based on a cascade of SPDT switches that feed the OFF-state signal for a certain connection between an RF chain and an antenna, to the line linking the same RF chain to the next antenna element of the array. We also employ 1-bit PSs to set either positive or negative real precoding matrix entries. Moreover, the line feeding the OFF-state signal incorporates another 1-bit PSs enabling the desired flexibility for the analog precoding matrix. In spite of that, ILs are equal to those in the analog network of [4] since the 1-bit PSs are connected in parallel. As a counterpart, the proposed analog network is slightly more complicated because of the additional PS and adder for each connection among antennas and RF chains.

In order to exploit the features of this new scheme, we propose an algorithm to design the pulse durations and pulse time delays of the switches, as well as the phases of the 1-bit PSs. An additional effect of the entry-independent pulse time delays is the entry-wise phase shifts on the analog precoding matrices of the harmonic replicas. As a consequence, the structure designed for the analog precoding matrix on the fundamental mode is destroyed on the precoding matrices corresponding to the harmonic replicas. Therefore, the received SR power in the harmonic frequencies is mitigated.

Due to the number of users supported by massive MIMO and the reduced number of RF chains in hybrid precoding, user scheduling is a key step prior to precoding design [10]-[12]. The limitation of the proposed TMA analog network to only allow real-valued analog precoders leads to a large consumption of degrees of freedom when Zero-Forcing (ZF) solutions are employed to handle inter-user interference. Therefore, a more flexible and careful scheduling strategy is necessary, like the angular scheduler proposed in this work. Our scheduler employs the more convenient angular directions of each user, taking into account the characteristic patterns of the analog network employed. Furthermore, the proposed algorithm evaluates the interference caused to previously scheduled users by using Maximum Ratio Trasmiter (MRT) real-valued analog precoders. Hence, the algorithm steps present low computational costs and the posed scheduler is very efficient. Further, with a slight adaptation, the procedure can be applied to nonTMA solutions for hybrid precoding.

Finally, we formulate a problem to determine the analog precoding vectors that can be recast to a Second-Order Cone Program (SOCP). Under this framework, it is possible to take into account the power spread in the harmonic frequencies and mitigate the SR. Computer experiments conducted in a broad variety of scenarios show the excellent performance results obtained with the proposed methods, compared to existing analog precoding approaches like [13], [14].

The contributions of this work are summarized as follows:

- A novel flexible analog network presenting an approximate OFF-state efficiency about $100 \%$, as well as an algorithm to set the analog processing network.

- An angular scheduler that takes into account the special features of the underlying analog network. Moreover, the inter-user interference is analyzed in a way that allows for a satisfactory scheduling policy with very low computational complexity.

- An analog precoding matrix problem formulation including the SR, resulting in remarkable achievable sum rates and power efficiencies over $90 \%$.

\section{SYSTEM MODEL}

Fig. 2 shows three different types of analog networks for hybrid digital-analog precoding. They are all fully connected networks [15], since there are $N_{\mathrm{RF}} M$ connections between the $N_{\mathrm{RF}}$ chains and the $M$ antennas, with $N_{\mathrm{RF}} \ll M$. Fig. 2 shows (a) the conventional analog network with high-resolution PSs; (b) the TMA analog network with SPST switches and 1-bit PSs considered in [4]; and (c) the proposed TMA analog network with SPDT switches.

The advantage of analog precoding with SPDT switches in (c) is that it does not suffer from the large ILs of the PSs in (a) [16], while it does not waste power during the OFF-state of the SPST switches in (b). Indeed, in the analog network with SPDT switches (c), the signals feeding the transmitting antennas result from the sum of the outputs of two SPDT switches. Accordingly, during the OFF-state, the power not employed by the $m$-th antenna is transferred to antenna $m+1$, except in the case of the last antenna $M$ where the connections with the RF chains are carried out with SPST switches. These are the only switches that fruitlessly absorb power power during the OFF-state.

Finally, note that all the switches throws are followed by a 1-bit PS to allow changing the sign of the corresponding line. The ILs of these simple PSs, however, are significantly lower than those of high-resolution PSs [4], [15].

\section{A. Signal Model and Problem Formulation}

We consider a wireless downlink where a Base Station (BS) with $M$ antennas sends data to $K$ single-antenna users. Since the BS is implemented with a reduced number of RF chains, only a subset of scheduled users $\mathcal{S}$, with $|\mathcal{S}| \leq N_{\mathrm{RF}}$, are simultaneously served. The source data vector $s \in \mathbb{C}^{|\mathcal{S}|}$ represents the input data to be sent to all users at a given time instant. Prior to its transmission, $s$ is precoded with the hybrid digital-analog architecture depicted in Fig. 1 and Fig. 2(c). First, the source data vector inputs the digital precoder $\boldsymbol{W}_{\mathrm{D}} \in \mathbb{C}^{N_{\mathrm{RF}} \times|\mathcal{S}|}$. Next, the digitally precoded signals enter the analog part represented by $\boldsymbol{W}_{\mathrm{A}} \in \mathcal{W}$ of size $\mathbb{R}^{M \times N_{\mathrm{RF}}}$ where $\mathcal{W}$ is conditioned by the analog network in Fig. 2(c) (see Sec. III-A).

After transmission, the received signal for the $k$-th user reads as

$$
y_{k}=\boldsymbol{h}_{k}^{\mathrm{H}} \boldsymbol{W}_{\mathrm{A}} \sum_{i \in \mathcal{S}} \boldsymbol{w}_{\mathrm{D}, i} s_{i}+n_{k},
$$

where $\boldsymbol{h}_{k} \in \mathbb{C}^{M}$ is the channel vector, $\boldsymbol{w}_{\mathrm{D}, i}$ is the $i$-th column of $\boldsymbol{W}_{\mathrm{D}}, s_{i}$ is the $i$-th user source symbol, and $n_{k}$ is the additive Gaussian noise $n_{k} \sim \mathcal{N}_{\mathbb{C}}\left(0, \sigma^{2}\right)$.

The performance metric considered is the achievable sum rate. Under the common assumptions of zero-mean uncorre- 


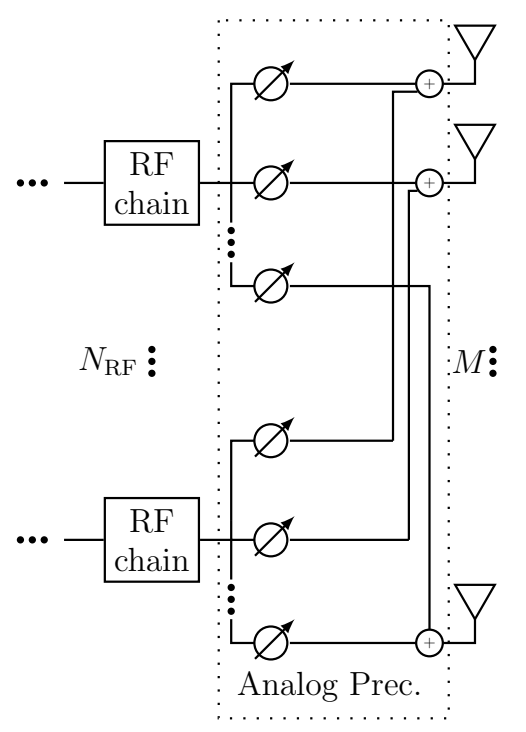

(a)

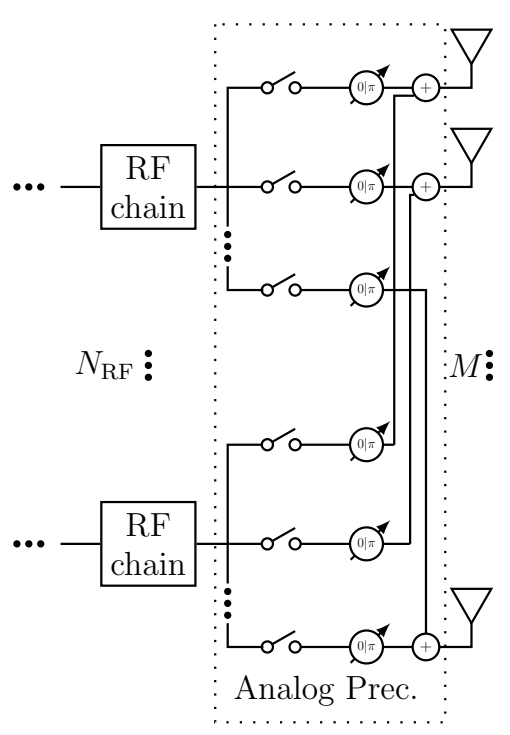

(b)

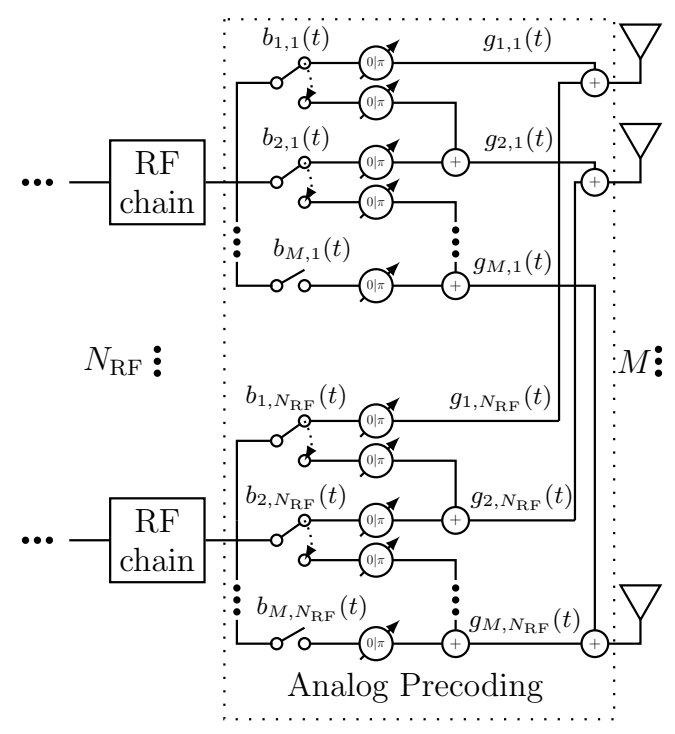

(c)

Fig. 2: Three types of analog networks for hybrid precoding: (a) Conventional with high-resolution PSs, (b) TMA with SPST switches and 1-bit PSs [4], (c) Proposed TMA with SPDT switches and 1-bit PSs.

lated Gaussian data signals, and considering user interference as noise, this metric reads as

$$
R=\sum_{k=1}^{K} R_{k}=\sum_{k=1}^{K} \log \left(1+\frac{\left|\boldsymbol{h}_{k}^{\mathrm{H}} \boldsymbol{W}_{\mathrm{A}} \boldsymbol{w}_{\mathrm{D}, k}\right|^{2}}{\sigma^{2}+\sum_{i \neq k}\left|\boldsymbol{h}_{k}^{\mathrm{H}} \boldsymbol{W}_{\mathrm{A}} \boldsymbol{w}_{\mathrm{D}, i}\right|^{2}}\right)
$$

Our objective is to determine $\mathcal{S}, \boldsymbol{W}_{\mathrm{A}}$ and $\boldsymbol{W}_{\mathrm{D}}$ that maximize (2). Due to the limitations imposed by the analog network, the problem is formulated as

$$
\max _{\mathcal{S}, \boldsymbol{W}_{\mathrm{A}} \in \mathcal{W}, \boldsymbol{W}_{\mathrm{D}}} \sum_{i \in \mathcal{S}} R_{i} \quad \text { s.t. } \quad\left\|\boldsymbol{W}_{\mathrm{A}} \boldsymbol{W}_{\mathrm{D}}\right\|_{\mathrm{F}}^{2} \leq P,
$$

where $P$ is the available transmit power. In order to successfully address this difficult combinatorial problem, we divide it into two subproblems. First, we obtain the set of scheduled users $\mathcal{S}$ (Sec. IV) and, next, we determine the hybrid precoder $\left\{\boldsymbol{W}_{\mathrm{A}}, \boldsymbol{W}_{\mathrm{D}}\right\}$ for the obtained set $\mathcal{S}(\mathrm{Sec} . \mathrm{V})$.

\section{B. Channel model}

We assume a Uniform Linear Array (ULA) at the transmitter so that the steering vectors $\boldsymbol{a}\left(\vartheta_{l}\right)$ have the form $\boldsymbol{a}\left(\vartheta_{l}\right)=\frac{1}{\sqrt{M}}\left[1, \mathrm{e}^{-\mathrm{j} \frac{2 \pi}{\lambda} d \cos \vartheta_{l}}, \ldots, \mathrm{e}^{-\mathrm{j} \frac{2 \pi}{\lambda} d(M-1) \cos \vartheta_{l}}\right]^{\mathrm{T}}$, where $\vartheta_{l} \in[0, \pi]$ is the Angle of Departure (AoD) for the $l$-th channel propagation path, $d$ is the distance between two consecutive array elements, and $\lambda$ represents the carrier wavelength. In this work, we will consider $d=\lambda / 2$. According to the channel model in [17], the channel vector $\boldsymbol{h}_{k}$, corresponding to the $k$-th user, is the superposition of $L_{k}$ propagation paths

$$
\boldsymbol{h}_{k}=\sum_{l=1}^{L_{k}} \boldsymbol{a}\left(\vartheta_{l, k}\right) \alpha_{l, k}
$$

where $\alpha_{l, k}$ are the complex gains associated to each path. We assume that gains for different paths are statistically independent and follow a normal distribution.

\section{Analog Network Setting}

TMAs are implemented with switches governed by periodic rectangular pulses [4], [5]. The SPDT switch between the $n$-th RF chain and the $m$-th antenna is modeled by the pulse waveform $p_{m, n}(t)$, that is periodically repeated every $T_{0}$, the TMA fundamental period. Moreover, the canonical pulses $p_{m, n}(t)$ are rectangular, symmetric, i.e., $p_{m, n}(t)=p_{m, n}(-t)$, and with unit-amplitude [5]. In the proposed scheme, the two throws of the SPDT switches are modeled by the pulse waveforms $b_{m, n}(t)$ and $1-b_{m, n}(t)$, respectively, where $b_{m, n}(t)$ is a delayed version of $p_{m, n}(t)$ with duration $\tau_{n, m}^{b} \in\left[0, T_{0}\right]$, i.e., $b_{m, n}(t)=p_{m, n}\left(t-\nu_{m, n}^{b}\right)$ where $\nu_{m, n}^{b}$ is the time delay.

As shown in Fig. 2(c), we utilize a 1-bit PS with phase $\phi_{m-1 \rightarrow m, n}$ to connect the lower output of the $m-1$ SPDT with the adder of the $(n, m)$ connection, and a 1-bit PS with phase $\phi_{m, n}$ to connect the upper throw of the $m$ SPDT to such adder. Hence, we eventually produce the combined pulses $g_{m, n}(t)$ as follows

$$
g_{m, n}(t)=\left(1-b_{m-1, n}(t)\right) \mathrm{e}^{\mathrm{j} \phi_{m-1 \rightarrow m, n}}+b_{m, n}(t) \mathrm{e}^{\mathrm{j} \phi_{m, n}} .
$$

The resulting time variant analog precoding matrix $\boldsymbol{W}_{\mathrm{A}}(t)$ comprises the functions $g_{m, n}(t)$ corresponding to the $N_{\mathrm{RF}} M$ connections among the RF chains and the antennas. Due to the periodic nature of the functions $g_{m, n}(t)$, each entry of $\boldsymbol{W}_{\mathrm{A}}(t)$ can be represented by its Fourier series. Indeed, the proposed scheme based on TMAs inherently generates replicas of the transmitted signal at the harmonic frequencies $\omega_{c}+q \omega_{0}$, with $q \in \mathbb{Z}, \omega_{0}=\frac{2 \pi}{T_{0}}$, and $\omega_{c}$ being the carrier frequency [5].

The entry-wise representation of $\boldsymbol{W}_{\mathrm{A}}(t)$ using Fourier series leads to a more convenient frequency dependent notation. 
Thus, for the harmonic mode $q$ we obtain

$$
\boldsymbol{W}_{\mathrm{A}}^{q}=\left[\begin{array}{ccc}
G_{1,1}^{q} & \ldots & G_{1, N_{\mathrm{RF}}}^{q} \\
\vdots & \ddots & \vdots \\
G_{M, 1}^{q} & \cdots & G_{M, N_{\mathrm{RF}}}^{q}
\end{array}\right],
$$

where each matrix entry is computed as [7], [18]

$$
G_{m, n}^{q}=\frac{1}{T_{0}} \int_{-\frac{T_{0}}{2}}^{\frac{T_{0}}{2}} g_{m, n}(t) \mathrm{e}^{-j q \omega_{0} t} d t .
$$

In this work, we will employ the fundamental mode $(q=0)$ to transmit data, while the remaining harmonic replicas will be considered undesired SR. Observe that the expression in (5) clearly separates the useful signal from the SR. Therefore, we aim at finding the analog precoder $\boldsymbol{W}_{\mathrm{A}}^{0}$ that maximizes the achievable sum rate in (3).

\section{A. Feasible Analog Precoders}

In this section we characterize the feasible set of analog precoders $\mathcal{W}$, such that $\boldsymbol{W}_{\mathrm{A}}^{0} \in \mathcal{W}$. One of the key features of the analog precoder with SPDT switches is its ability to obtain any value for $G_{m, n}^{0}$ within the interval $\in[-1,1]$. This feasible space for analog precoding is the same as in [4]. Contrary to [4], the pulse durations of all the SPDT switches associated to the $n$-th RF chain have to be jointly determined due to the cascade structure of the analog network. Consequently, we seek for a method to set the pulse durations $\tau_{m, n}^{b}$, the delays $\nu_{m, n}^{b}$, and the phase shifts $\phi_{m-1 \rightarrow m, n}$ and $\phi_{m, n}$, to attain the desired gains $G_{m, n}^{0}$ for the $n$-th RF chain.

We provide an algorithmic solution where the switching state changes only once within $T_{0}$, i.e., it does not require faster SPDT switches. Moreover, we bound the pulse amplitude such that $|g(t)| \leq 1$. Finally, to simplify the computation of (6) we ensure that the pulses can be written as $g_{m, n}(t)=$ $p_{m, n}\left(t-\nu_{m, n}^{g}\right)$, that is, the unit-amplitude symmetric pulse with a delay $\nu_{m, n}^{g}$ and a duration $\tau_{m, n}^{g}$. Two examples of this equality are shown in Figs. 3 and 4. Under the former conditions, we compute the Fourier coefficients as

$$
\begin{aligned}
G_{m, n}^{q} & =\frac{1}{T_{0}} \int_{-\frac{T_{0}}{2}}^{\frac{T_{0}}{2}} g_{m, n}(t) \mathrm{e}^{-j q \omega_{0} t} d t \\
& =\frac{1}{T_{0}} \operatorname{sign}\left(G_{m, n}^{0}\right) \int_{-\frac{T_{0}}{2}}^{\frac{T_{0}}{2}} p_{m, n}\left(t-\nu_{m, n}^{g}\right) \mathrm{e}^{-j q \omega_{0} t} d t \\
& =\operatorname{sign}\left(G_{m, n}^{0}\right)\left|G_{m, n}^{0}\right| \operatorname{sinc}\left(\pi q\left|G_{m, n}^{0}\right|\right) \mathrm{e}^{-j q \omega_{0} \nu_{m, n}^{g}},
\end{aligned}
$$

where $\left|G_{m, n}^{0}\right|=\frac{\tau_{m, n}^{g}}{T_{0}}$ are the normalized TMA pulse durations.

Alg. 1 summarizes the procedure to set the analog network. Consider a certain RF chain $n$. Line 3 determines the difference between the OFF-state duration of the SPDT switch associated to the antenna $m-1$ and the desired pulse duration for the $m$-th antenna, $\tau_{m, n}^{g}=\left|G_{m, n}^{0}\right| T_{0}$. Next, we set the sign of the 1-bit PS of the cascade connection according to the desired $G_{m, n}^{0}$ in line 4 . Then, lines 5 to 13 increase or reduce the duration of the pulse $m-1$ to achieve $\tau_{m, n}^{g}$, and determine the time shifts $\nu_{m, n}^{b}$ and $\nu_{m, n}^{g}$ such that the aforementioned conditions on $g_{m, n}(t)$ are achieved. As a
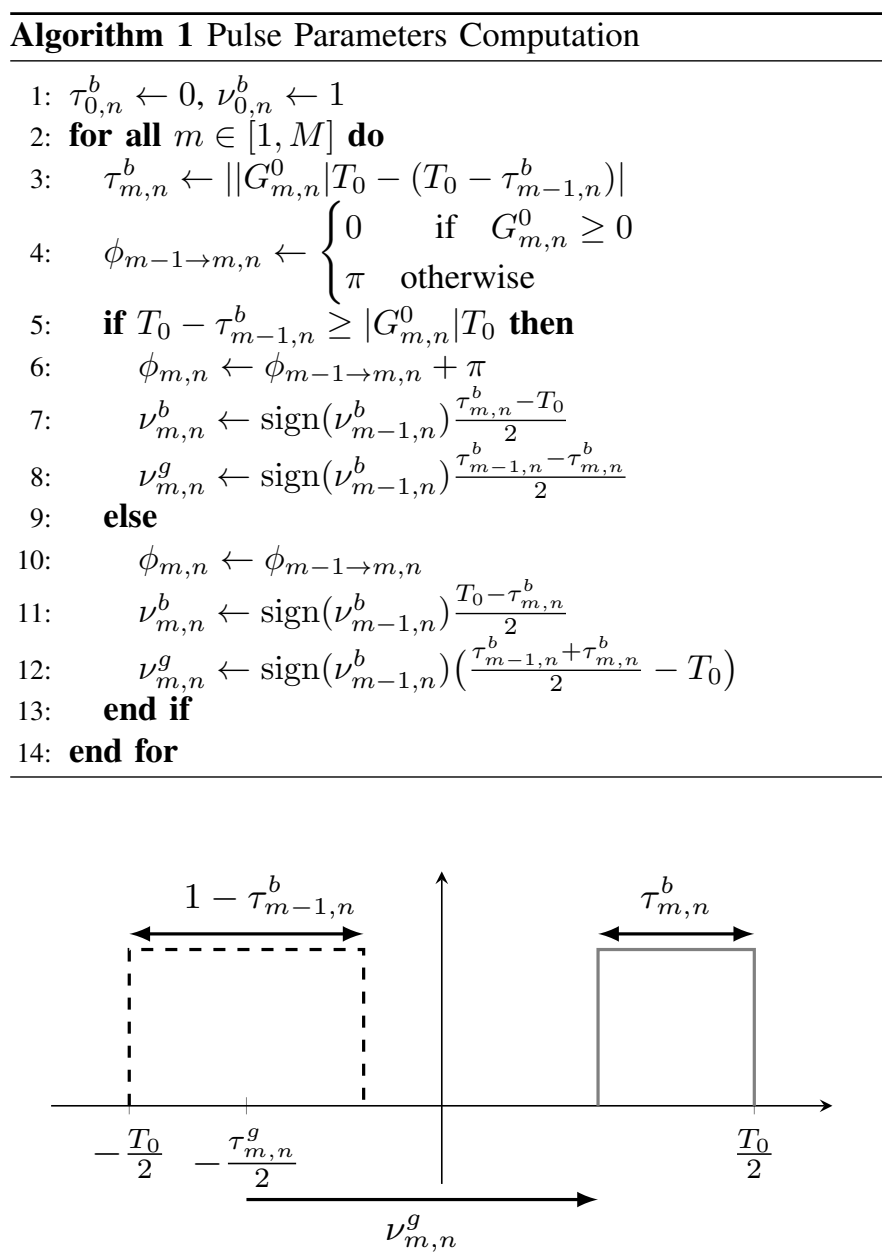

Fig. 3: Example of SPDT switch configuration to achieve the desired pulse length $\tau_{m, n}^{g}$ reducing off-state the duration from the line $(m-1, n)$.

result, we obtain the $n$-th column of $\boldsymbol{W}_{\mathrm{A}}^{0}$. The algorithm is repeated for $n=\left\{1, \ldots, N_{\mathrm{RF}}\right\}$.

To simplify the analysis, it is possible to relax the constraint $\boldsymbol{W}_{\mathrm{A}}^{0} \in \mathcal{W}$ and only require for the analog precoding matrix entries to be real. This relaxation is transparent to the overall hybrid precoder, as its effects are easily compensated with the baseband counterpart (see Sec. VI). Henceforth, we will consider that $\boldsymbol{W}_{\mathrm{A}}^{0} \in \mathbb{R}^{M \times N_{\mathrm{RF}}}$.

\section{B. TMA Power Losses}

The expression in (7) is also useful to obtain a relationship between the analog precoders at the $q$ harmonic and the fundamental mode. Since $G_{m, n}^{q}$ for $q \neq 0$ in (7) is an element-wise phase rotation and module reduction of $G_{m, n}^{0}$, we can establish a simple relationship among the analog precoding matrix for the different harmonics using the Hadamard product $\boldsymbol{W}_{\mathrm{A}}^{q}=$ $\boldsymbol{W}_{\mathrm{A}}^{0} \odot \boldsymbol{S}^{q}$, with $\left[\boldsymbol{S}^{q}\right]_{m, n}=\operatorname{sinc}\left(\pi q\left|\left[\boldsymbol{W}_{\mathrm{A}}^{0}\right]_{m, n}\right|\right) \mathrm{e}^{-j q \omega_{0} \nu_{m, n}}$. Next, note that the total transmit power is the one radiated over all the harmonics, i.e.,

$$
P_{\mathrm{TX}}=\sum_{q=-\infty}^{\infty}\left\|\boldsymbol{W}_{\mathrm{A}}^{q} \boldsymbol{W}_{\mathrm{D}}\right\|_{\mathrm{F}}^{2} .
$$




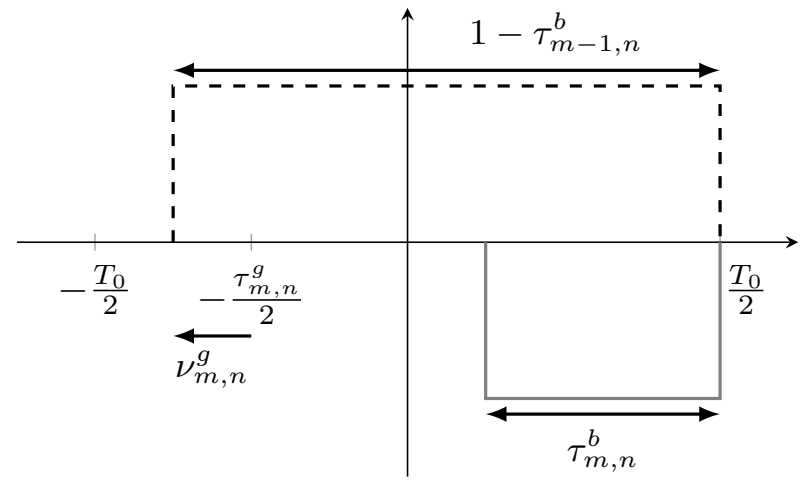

Fig. 4: Example of SPDT switch configuration to achieve the desired pulse length $\tau_{m, n}^{g}$ increasing the off-state duration from the line $(m-1, n)$.

From (8), we define the transmit power efficiency as

$$
\eta_{\mathrm{TX}}=\frac{\left\|\boldsymbol{W}_{\mathrm{A}}^{0} \boldsymbol{W}_{\mathrm{D}}\right\|_{\mathrm{F}}^{2}}{P_{\mathrm{TX}}} .
$$

Besides the power loss, SR makes difficult the transmission of wideband signals with bandwidths larger than $2 \omega_{0}$ [4]. In addition, it causes interference to other systems working in frequency bands containing the harmonic frequencies $\omega_{c}+$ $q \omega_{0}$. To evaluate this effect, we introduce the received power efficiency

$$
\eta_{\mathrm{RX}}=\frac{\left\|\boldsymbol{H}^{\mathrm{H}} \boldsymbol{W}_{\mathrm{A}}^{0} \boldsymbol{W}_{\mathrm{D}}\right\|_{\mathrm{F}}^{2}}{\sum_{q=-\infty}^{\infty}\left\|\boldsymbol{H}^{\mathrm{H}} \boldsymbol{W}_{\mathrm{A}}^{q} \boldsymbol{W}_{\mathrm{D}}\right\|_{\mathrm{F}}^{2}},
$$

with $\boldsymbol{H}$ comprising the channels for the scheduled users. This interference is mitigated by using the proposed analog network. Note that, for $q \neq 0$, the received power generally decreases due to the two factors in $\left[\boldsymbol{S}^{q}\right]_{m, n}$. The first factor, $\operatorname{sinc}\left(\pi q\left|G_{m, n}^{0}\right|\right)$, leads to smaller matrix norms for the harmonics, $\left\|\boldsymbol{W}_{\mathrm{A}}^{q}\right\|_{\mathrm{F}}$, compared to that for the fundamental mode, $\left\|\boldsymbol{W}_{\mathrm{A}}^{0}\right\|_{\mathrm{F}}$. Moreover, the unitary rotations $\mathrm{e}^{-j q \omega_{0} \nu_{m, n}^{g}}$ introduced using Alg. 1 remove the structural properties from $\boldsymbol{W}_{\mathrm{A}}^{0}$. Hence, the interference power in the harmonic frequencies $\left\|\boldsymbol{H}^{\mathrm{H}} \boldsymbol{W}_{\mathrm{A}}^{q} \boldsymbol{W}_{\mathrm{D}}\right\|_{\mathrm{F}}^{2}$ is generally small. Nevertheless, the lack of an analytic solution to $\nu_{m, n}^{g}$, complicates the evaluation of the combination of the two factors in $\left[\boldsymbol{S}^{q}\right]_{m, n}$. Empirical results show that the received power in the harmonics is reduced employing the proposed method.

Other source of energy inefficiency in TMAs is the switching hardware. For any non-trivial analog precoding design, SPST switches will unavoidably remain in the OFF-state during a certain amount of time therefore absorbing the corresponding available energy. Following the discussion in [4], we analyze the hardware efficiency for the analog precoder with SPDT switches. Since the number of switching devices is $(M-1) N_{\mathrm{RF}}$ SPDTs and $N_{\mathrm{RF}}$ SPSTs, the OFF-state efficiency reads as

$$
\eta_{\mathrm{OFF}}=\frac{(M-1) N_{\mathrm{RF}}+\sum_{n=1}^{N_{\mathrm{RF}}}\left|G_{M, n}^{0}\right|}{M N_{\mathrm{RF}}} .
$$

Since $N_{\mathrm{RF}} \ll M$, the OFF-state efficiency of the scheme in Fig. 2(c) is $\eta_{\mathrm{OFF}} \approx 1$.

\section{Angular Scheduling}

The optimization problem (3) is a difficult one. In order to solve it satisfactorily, we divide it in two parts. First, we obtain the set of scheduled users $\mathcal{S}$ and, next, we determine the hybrid precoder $\left\{\boldsymbol{W}_{\mathrm{A}}, \boldsymbol{W}_{\mathrm{D}}\right\}$ for the obtained set $\mathcal{S}$. The former subproblem, determining the set of scheduled users $\mathcal{S}$, is addressed in this section while the latter is considered in the ensuing section.

In the literature, different strategies for user scheduling have been proposed, although their applicability to hybrid precoding is rather limited due to the stringent hardware restrictions. Without prior knowledge, determining the set of active users is a combinatorial problem. However, greedy approaches have shown excellent performance [19]. Hereafter, we will follow this approximation.

\section{A. Limitation of ZF Scheduling Policies}

Most existing scheduling policies rely on interference cancellation [19], [20]. It is well known that the ZF strategy is asymptotically optimal in the high SNR regime [21]. This fact has motivated its adaptation to hybrid precoding [12], [14]. ZF computes the precoder for a certain user in the nullspace of the matrix comprising the channels of the rest of the users. Nevertheless, using the analog network based on SPDT switches, including a certain user in the scheduler reduces the interference free subspace for the remaining users faster than in the case of full digital precoding.

Let us consider that user $k$ is selected by the scheduler. Then, the ZF condition holds for the feasible precoding subspace spanned by the basis

$$
\boldsymbol{U}=\mathbf{I}-\boldsymbol{\Pi}=\mathbf{I}-\frac{\boldsymbol{h}_{k} \boldsymbol{h}_{k}^{\mathrm{H}}}{\left\|\boldsymbol{h}_{k}\right\|_{\mathrm{F}}^{2}} .
$$

Therefore, the precoder $\boldsymbol{w}_{\mathrm{ZF}}$ for the user $i \neq k$ that maximizes the SNR under the ZF condition, satisfies $\boldsymbol{w}_{\mathrm{ZF}}^{\mathrm{H}} \boldsymbol{\Pi}=\mathbf{0}$. Recall now that in our proposed analog network entries in $\boldsymbol{w}_{\mathrm{ZF}}$ are real valued. Then, splitting up the projector as $\boldsymbol{\Pi}_{R}=\operatorname{Re}(\boldsymbol{\Pi})$ and $\boldsymbol{\Pi}_{I}=\operatorname{Im}(\boldsymbol{\Pi})$ the ZF condition results in

$$
\boldsymbol{w}_{\mathrm{ZF}}^{\mathrm{T}}\left(\boldsymbol{\Pi}_{R}+\mathrm{j} \boldsymbol{\Pi}_{I}\right)=\mathbf{0} .
$$

Note that it is not possible to find a feasible $\boldsymbol{w}_{\mathrm{ZF}}$ such that $\boldsymbol{w}_{\mathrm{ZF}}^{\mathrm{T}} \boldsymbol{\Pi}_{R}=-\mathrm{j} \boldsymbol{w}_{\mathrm{ZF}}^{\mathrm{T}} \boldsymbol{\Pi}_{I}$. Therefore, $\boldsymbol{w}_{\mathrm{ZF}}$ must lie in the intersection of the nullspaces of the real and complex channel subspaces, i.e., $\boldsymbol{w}_{\mathrm{ZF}}^{\mathrm{T}} \boldsymbol{\Pi}_{R}=\boldsymbol{w}_{\mathrm{ZF}}^{\mathrm{T}} \boldsymbol{\Pi}_{I}=\mathbf{0}$. Thus, the feasible subspace for precoding reduces up to twice as fast as in the complex-valued case. Therefore, the number of users that can be served simultaneously by the BS satisfying the ZF condition is generally smaller than for digital precoding.

\section{B. MRT Real Precoders}

The MRT precoder maximizes the signal strength for the steering vector of a certain angular direction. However, the limitations imposed by the proposed analog network also affect 
the design of the MRT. Indeed, for the target angle $\vartheta$, MRT is the solution to the following optimization problem

$$
\boldsymbol{w}_{\mathrm{MRT}}(\vartheta)=\underset{\boldsymbol{w} \in \mathbb{R}}{\operatorname{argmax}}\left|\boldsymbol{w}^{\mathrm{T}} \boldsymbol{a}(\vartheta)\right| \quad \text { s.t. } \quad\|\boldsymbol{w}\|_{2}=1,
$$

that is, $\boldsymbol{w}_{\text {MRT }}(\vartheta)=\boldsymbol{u}_{\max }\left(\operatorname{Re}\left\{\boldsymbol{a}(\vartheta) \boldsymbol{a}^{\mathrm{H}}(\vartheta)\right\}\right)$ with $\boldsymbol{u}_{\max }(\boldsymbol{X})$ the eigenvector corresponding to the maximum eigenvalue of $\boldsymbol{X}$. This is immediate from the observation that $\boldsymbol{w}^{\mathrm{T}} \operatorname{Im}\left\{\boldsymbol{a}(\vartheta) \boldsymbol{a}^{\mathrm{H}}(\vartheta)\right\} \boldsymbol{w}=0$ for any feasible $\boldsymbol{w}$.

Conventional complex MRT precoders are computationally very efficient. This is especially relevant to reduce the computational burden of user schedulers. Thus, to realize a similar property for the real case, we pose to employ the precoder $\boldsymbol{w}_{R}(\vartheta)$ given by

$$
\boldsymbol{w}_{R}(\vartheta)=\frac{\boldsymbol{a}_{R}(\vartheta)}{\left\|\boldsymbol{a}_{R}(\vartheta)\right\|_{2}},
$$

with $\boldsymbol{a}_{R}(\vartheta)=\operatorname{Re}\{\boldsymbol{a}(\vartheta)\}$. Note that $\boldsymbol{w}_{R}(\vartheta)$ is asymptotically optimal to (14) (see Appendix A). For the array sizes considered in this work, $M \approx 100,(15)$ is near-optimal for a wide range of angular directions.

\section{Proposed Angular Scheduler}

An insightful interpretation of the limitation discussed in Sec. IV-A is reached by considering the symmetry of the radiation pattern for real analog precoders. Note that pointing the precoder to $\vartheta$, with $\vartheta<\frac{\pi}{2}$, produces also a symmetric beam pointing to $\pi-\vartheta$. Therefore, the additional interference generated must be considered at the scheduling stage. This symmetry, however, might be profitably exploited in Non Lineof-sight (NLOS) scenarios if two channel propagation paths impinge the array with angular directions close to $\vartheta$ and $\pi-\vartheta$.

Contrary to conventional schedulers that consider the channel vector, the aforementioned particularities of real analog precoding motivate us to determine the most promising channel angular directions. This subtler procedure enables the possibility of selecting channel paths offering good gains and, at the same time, alleviates the excessive consumption of degrees of freedom discussed in Sec. IV-A. The set $\mathcal{S}$ will be thereby determined by the users whose channels contain the selected angles.

We pose to select the angles in a greedy fashion using a variation of the Simultaneous-Orthogonal Matching Pursuit (SOMP) algorithm proposed in [22], which is a generalization of OMP where Gram-Schmidt is performed at each iteration [23]. This method performs simultaneous sparse recovery by greedily selecting at each iteration a measurement vector from a predefined set of candidates. The metric is next adapted as a function of the previously selected measurement vectors. Following a similar strategy, some of the schedulers in the literature select the user with the most promising channel, and use a Gram-Schmidt procedure to update the metric computing the projector into the nullspace of the selected channel [19]. In our setup, this search stage is performed over the set of AoDs for all users, and the selection rule at iteration $n$ is

$$
\vartheta_{l, k}=\underset{\vartheta_{j, i} \in \Omega^{(n)}}{\operatorname{argmax}} f^{(n)}\left(\vartheta_{j, i}\right),
$$

where $\Omega^{(n)}$ is a set containing the eligible angles and $f^{(n)}(\cdot)$ is the metric to maximize at the $n$-th iteration. Unfortunately, the above mentioned Gram-Schmidt procedure does not apply due to the real nature of the analog precoders for the proposed architecture. In other words, it is not possible to build a basis for the interference-free subspace for precoding using a real projection matrix. Then, a different metric $f^{(n)}(\cdot)$ is necessary for the considered setup.

1) Proposed Metric: Our heuristic consists in selecting the angular direction in the dual uplink [24] that provides larger equivalent channel gains but considering the interference caused to previously scheduled users. Then, for the $j$-th path of the $k$-th user, the metric at iteration $n$ reads as

$$
f^{(n)}\left(\vartheta_{j, k}\right)=\frac{\left|\boldsymbol{w}_{\mathrm{MRT}}^{\mathrm{T}}\left(\vartheta_{j, k}\right)\left(\boldsymbol{h}_{k}-\sum_{\vartheta_{l, k} \in \Upsilon^{(n)}} \boldsymbol{a}\left(\vartheta_{l, k}\right) \alpha_{l, k}\right)\right|^{2}}{\beta+\sum_{i \in \mathcal{S}^{(n)}, i \neq k}\left|\boldsymbol{w}_{\mathrm{MRT}}^{\mathrm{T}}\left(\vartheta_{j, k}\right) \boldsymbol{h}_{i}\right|^{2}},
$$

where $\mathcal{S}^{(n)}$ is the set of scheduled users at the $n$-th iteration, and $\Upsilon^{(n)}$ the sequence of selected angles at the $n$-th iteration. This metric is similar to the Signal-to-Interference-plus-Noise Ratio (SINR) employed for Successive Interference Cancellation (SIC) [25]. However, the numerator of (17) evaluates the gain of pointing the real MRT precoder towards the $j$ th path angular direction, $\vartheta_{j, k}$, but removes the contribution of the paths already considered for user $k$. In addition, the parameter $\beta$ in the denominator of (17) determines the sensitivity of the metric to the inter-user interference, approaching zero-interference and SNR maximization for small and large values of $\beta$, respectively. Observe that evaluating the metric employing the real MRT includes the effects resulting from the symmetry of the radiation pattern.

To establish the AoDs priority, we define the sequence $\Theta^{(n)} \supseteq \Upsilon^{(n)}$ as

$$
\begin{aligned}
\Theta^{(n)}= & \left\{\vartheta_{l_{1}, k_{1}}, \ldots, \vartheta_{l_{N}, k_{N}} \mid k_{i} \in \mathcal{S}^{(n)} \forall i,\right. \\
& \left.\delta_{l_{1}, k_{1}} \geq \ldots \geq \delta_{l_{N}, k_{N}}\right\} .
\end{aligned}
$$

Observe that $\Theta^{(n)}$ contains the AoDs ordered according to $\delta_{l, k}$, i.e., the value of the metric for the angle associated to the $l$-path of the scheduled user $k$. The total number of paths is denoted as $N=\sum_{i \in \mathcal{S}^{(n)}} L_{i}$.

2) Proposed Algorithm: Alg. 2 summarizes the greedy selection procedure. In line 4 , the proposed algorithm selects the angle $\vartheta_{l, k}$ that maximizes the metric $f^{(n)}(\cdot)$. Recall that $f^{(n)}(\cdot)$ in (17) only considers the interference caused to previously scheduled users. Consequently, the metric value obtained for a given angle changes at each iteration $n$. Nevertheless, our greedy procedure evaluates the metric for $\vartheta_{l, k}$ only once and the resulting value is stored in $\delta_{l, k}$ (line (6)). This decision prioritizes the already scheduled users since $f^{(n)}(\cdot)$ strongly penalizes inter-user interference for increasing iteration number $n$. Next, all the angles for such user are removed from the set of eligible angles for the next iteration $\Omega^{(n+1)}$. Moreover, user $k^{(n)}$ is included in the set of scheduled users $\mathcal{S}^{(n+1)}$. Then, the sequence $\Theta^{(n+1)}$ is updated such that it contains all the angles for the users belonging to the set $\mathcal{S}^{(n+1)}$ ordered according to its metric values. Afterwards, $f^{(n+1)}(\cdot)$ is updated according to the current choice $\vartheta_{l, k}$. The 


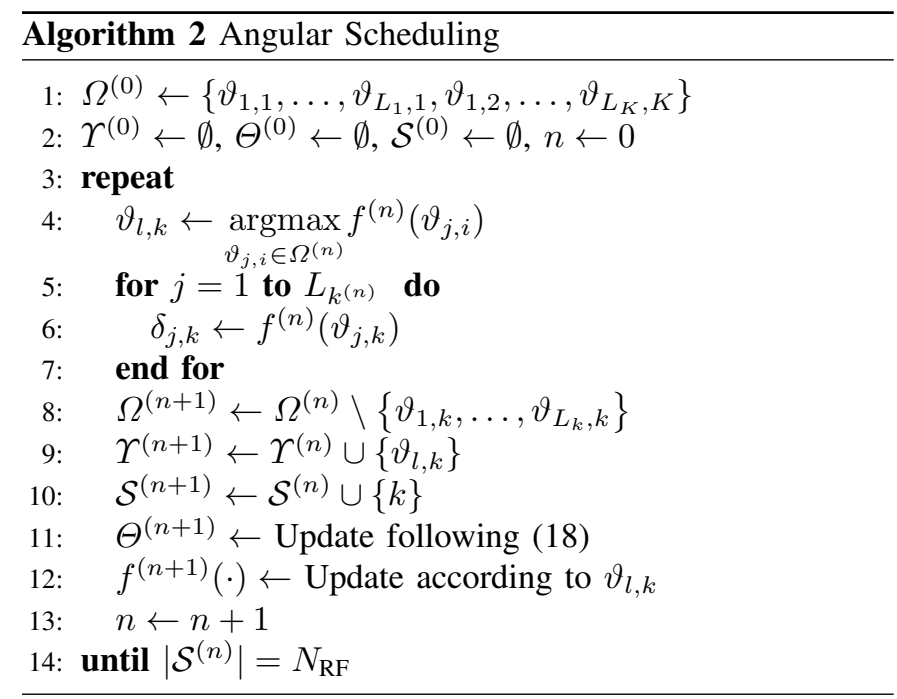

algorithm terminates when the number of scheduled users is equal to $N_{\mathrm{RF}}$.

\section{Low Complexity Angular Scheduling}

We now propose an efficient method to evaluate the metric employed by the scheduler. To this end, we characterize the signal power obtained when pointing to $\vartheta_{l}$ instead of to the target angular direction $\vartheta_{t}$. For completeness, we consider both complex and real scenarios. Thus, in addition to the analog network with SPDT switches, this strategy can also be applied to analog networks with PSs. Moreover, the real case can be analyzed using angular differences for complex-valued scenarios, as discussed in the following.

We start expressing the spatial response of $\boldsymbol{w}_{\mathrm{MRT}}\left(\vartheta_{l}\right)=$ $\boldsymbol{a}\left(\vartheta_{l}\right)$ towards the target angular direction $\vartheta_{t}$ as

$$
\boldsymbol{w}_{\mathrm{MRT}}^{\mathrm{H}}\left(\vartheta_{l}\right) \boldsymbol{a}\left(\vartheta_{t}\right)=\rho(\varepsilon)=\mathrm{e}^{\mathrm{j} \frac{(M-1) \pi}{2} \varepsilon} \frac{\sin \left(\frac{M \pi}{2} \varepsilon\right)}{M \sin \left(\frac{\pi}{2} \varepsilon\right)},
$$

with $\varepsilon=\cos \vartheta_{l}-\cos \vartheta_{t} \in[-2,2]$. Note that $\rho(\varepsilon)$ is periodic and symmetric around 0 , but not monotonic on $\varepsilon$. Moreover, the nulls and the local maximum of the function in $[-2,2]$ are located at $\varepsilon= \pm \frac{2 m}{M}$ and $\varepsilon \approx \pm \frac{2 n+1}{M}$, respectively, with $m \in\{1,2, \ldots, M-1\}$ and $n \in\{1,2, \ldots, M-2\}$. Further, $\rho(\varepsilon) \approx \frac{1}{M \sin \left(\frac{\pi}{2} \varepsilon\right)}$ for the local maximum points. Regarding the angular distance $\left|\vartheta_{t}-\vartheta_{l}\right|$, the power attenuation is again periodic but not symmetric around 0 except for $\vartheta_{t}=\frac{\pi}{2}$ and $\vartheta_{t}=0$. Hence, in the interest of describing the attenuation, we consider the bound $\zeta$ and the angles $\vartheta_{L} \leq \vartheta_{t} \leq \vartheta_{U}$ satisfying

$$
\begin{aligned}
& \left\{\vartheta \mid \vartheta \leq \vartheta_{L}\right\} \Rightarrow\left|\boldsymbol{w}_{d}^{\mathrm{H}}\left(\vartheta_{t}\right) \boldsymbol{a}(\vartheta)\right| \leq \zeta, \\
& \left\{\vartheta \mid \vartheta_{U} \leq \vartheta\right\} \Rightarrow\left|\boldsymbol{w}_{d}^{\mathrm{H}}\left(\vartheta_{t}\right) \boldsymbol{a}(\vartheta)\right| \leq \zeta,
\end{aligned}
$$

for $\vartheta_{t}, \vartheta_{L}, \vartheta_{U} \in\left[0, \frac{\pi}{2}\right]$. Note that periodicity allows us to focus on this interval without loss of generality. If such angles $\vartheta_{L}$ and $\vartheta_{U}$ exist, they are computed as

$$
\begin{aligned}
& \vartheta_{L} \leq \arccos \left(\cos \vartheta_{t}+\frac{2}{\pi} \arcsin \left(\frac{1}{\zeta M}\right)\right), \\
& \vartheta_{U} \geq \arccos \left(\cos \vartheta_{t}-\frac{2}{\pi} \arcsin \left(\frac{1}{\zeta M}\right)\right) .
\end{aligned}
$$

The above expressions show that the target angles leading to precoders with maximum and minimum beam widths are located close to the edges of the considered interval, i.e., 0 and $\frac{\pi}{2}$, respectively. Accordingly, the feasible values of $\zeta$ are given by $\zeta=\max \left\{\zeta_{L}, \zeta_{U}\right\}$, with

$$
\zeta_{L} \geq \frac{1}{M \sin \left(\frac{\pi}{2}\left(1-\cos \vartheta_{t}\right)\right)}, \quad \zeta_{U} \geq \frac{1}{M \sin \left(\frac{\pi}{2} \cos \vartheta_{t}\right)} .
$$

Note that $\zeta_{L}$ and $\zeta_{U}$ do not apply on the lower and upper edges, respectively. These expressions are useful to determine reasonable bounds for the interference in the problem formulations of Sec. V-B, or to perform screening to speed up the angular scheduler procedure. Nevertheless, when considering real precoding vectors, equations (19)-(22) have to be adapted.

Unfortunately, the precoding vector in (14) does not lead to a closed-form expression for the interference due to the eigenvalue operation. Therefore, for the real case we propose to use instead the $\boldsymbol{w}_{R}\left(\vartheta_{t}\right)$ in (15) to obtain the following signal power attenuation

$$
\boldsymbol{w}_{R}^{\mathrm{T}}\left(\vartheta_{l}\right) \boldsymbol{a}\left(\vartheta_{t}\right)=\frac{1}{2\left\|\boldsymbol{a}_{R}\left(\vartheta_{l}\right)\right\|_{2}}(\rho(\varepsilon)+\rho(\epsilon)),
$$

with $\epsilon=-\cos \vartheta_{l}-\cos \vartheta_{t}$. Concerning the attenuation bound $\hat{\zeta}$ for this scenario, it is possible to obtain the angles $\vartheta_{L}$ and $\vartheta_{U}$ in (20) by bounding the envelope of (23) as

$$
\left|\boldsymbol{w}_{R}^{\mathrm{T}}\left(\vartheta_{t}\right) \boldsymbol{a}(\vartheta)\right| \leq \frac{1}{\left|M \sin \left(\frac{\pi}{2} \varepsilon\right)\right|\left\|\boldsymbol{a}_{R}\left(\vartheta_{l}\right)\right\|_{2}} .
$$

See App. B for the details. Notice that this bound is a scaled version of the envelope of $\rho(\varepsilon)$. Thus, a tighter bound is obtained by removing the vector norm from (24), since $\left|\boldsymbol{w}_{R}^{\mathrm{T}}\left(\vartheta_{t}\right) \boldsymbol{a}(\vartheta)\right| \leq\left|\boldsymbol{w}_{\mathrm{MRT}}^{\mathrm{H}}\left(\vartheta_{t}\right) \boldsymbol{a}(\vartheta)\right|$, with $\boldsymbol{w}_{\mathrm{MRT}}\left(\vartheta_{t}\right)$ the analog precoder in the complex case. Indeed, real analog precoders produce weaker inter-user interference.

Finally, according to the decomposition $\boldsymbol{h}=\sum_{l=1}^{L} \boldsymbol{a}\left(\vartheta_{l}\right) \alpha_{l}$, we evaluate products in (17) by using (23) as scalar functions,

$$
\left|\boldsymbol{w}_{\mathrm{MRT}}^{\mathrm{T}}\left(\vartheta_{t}\right) \boldsymbol{h}\right| \approx \frac{1}{2\left\|\boldsymbol{a}_{R}\left(\vartheta_{l}\right)\right\|_{2}}\left|\sum_{l=1}^{L} \alpha_{l}\left(\rho\left(\varepsilon_{l}\right)+\rho\left(\epsilon_{l}\right)\right)\right|,
$$

with $\varepsilon_{l}=\cos \vartheta_{l}-\cos \vartheta_{t}$, and $\epsilon_{l}=-\cos \vartheta_{l}-\cos \vartheta_{t}$. It is apparent that this approach dramatically reduces the computational complexity of the proposed scheduler.

The advantages of the proposed angular scheduler are summarized as follows: 1) it mitigates the inter-user interference, but allows the system to accommodate more users than ZF; 2) the scheduler presents a very high computational efficiency; and 3) the previous advantages are achieved for computationally cheap MRT analog precoders. Furthermore, this framework can be applied to other scenarios by adapting the selection rule to the considered architecture.

\section{TMA HYBRID PRECODING}

After the application of Alg. 2, a sequence of angles $\Theta^{(n)}$ and corresponding users $\mathcal{S}^{(n)}$ is provided for each iteration $n$. Then, the optimization problem (3) can be reduced to

$$
\max _{\boldsymbol{W}_{\mathrm{A}}^{0}, \boldsymbol{W}_{\mathrm{D}}} \sum_{i \in \mathcal{S}^{(n)}} R_{i} \quad \text { s.t. } \quad\left\|\boldsymbol{W}_{\mathrm{A}}^{0} \boldsymbol{W}_{\mathrm{D}}\right\|_{\mathrm{F}}^{2} \leq P .
$$


Next, the analog and baseband precoders are determined using an algorithmic solution according to the angular priorities. At each step $n$, our approach computes the hybrid precoding matrices and power allocation for $\Theta^{(n)}$, and evaluates the achievable throughput for this sequence. The iterative procedure terminates when including new channel paths, or angles of the sequence, does not provide higher achievable sum rates. In the following sections we detail the main steps of the proposed method.

\section{A. Computing Desired Precoding Directions}

A common approach to determine analog precoding matrices is to perform a search over a candidate set containing the MRT precoders for certain angles, that is,

$$
\boldsymbol{W}_{\mathrm{A}}=\left[\boldsymbol{w}_{\mathrm{MRT}}\left(\vartheta_{1}\right), \boldsymbol{w}_{\mathrm{MRT}}\left(\vartheta_{2}\right), \ldots, \boldsymbol{w}_{\mathrm{MRT}}\left(\vartheta_{N_{\mathrm{RF}}}\right)\right],
$$

see e.g. [3], [13], [14], [26]. This strategy assigns one angle per user and thus restricts the number of angles employed to $N_{\mathrm{RF}}$. A drawback of this scheme arises when dealing with channels $\boldsymbol{h}$ having $L>1$ propagation paths. By pointing to a single target direction $\vartheta_{t}$, associated with the complex gain $\alpha_{t}$, the power ratio w.r.t. digital precoding is given by

$$
\frac{\left|\boldsymbol{w}_{\mathrm{MRT}}^{\mathrm{H}}\left(\vartheta_{t}\right) \boldsymbol{h}\right|^{2}}{\|\boldsymbol{h}\|_{2}^{2}} \approx \frac{\left|\boldsymbol{w}_{\mathrm{MRT}}^{\mathrm{H}}\left(\vartheta_{t}\right) \boldsymbol{a}\left(\vartheta_{t}\right)\right|^{2}\left|\alpha_{t}\right|^{2}}{\sum_{l \neq t}\left|\alpha_{l}\right|^{2}},
$$

where the approximation is accurate for the common assumption of uniform distributed angles and practical numbers of antennas, $M \approx 100$, and holds with equality in the asymptotic regime. Note that the power loss reduces in Line-of-Sight (LoS) scenarios where $L$ is small and there is a dominant path. Conversely, the analog network with SPDT switches enables pointing to several angular directions.

Since the analog precoding vectors are flexible enough to cover several angular directions, we propose to design the analog precoding matrix using an iterative procedure. At each step $r$, we design a direction vector for the $r$-th RF chain, $\boldsymbol{d}_{r}^{(n)}$, by considering the angular directions obtained at the $n$ th iteration of Alg. 2, $\Theta^{(n)}$. When the sequence $\Theta^{(n)}$ assigns $I_{\text {end }}-I_{\text {ini }} \geq 1$ consecutive angles to user $k_{I_{\text {ini }}}$, with $I_{\text {ini }}$ and $I_{\text {end }}$ positions in the sequence, a single real precoding vector is enough to cover $I_{\text {end }}-I_{\text {ini }}$ angles, i.e.,

$$
\boldsymbol{d}_{r}^{(n)}=\sum_{i=I_{\mathrm{ini}}}^{I_{\mathrm{end}}} \boldsymbol{a}\left(\vartheta_{l_{i}, k_{i}}\right) g_{l_{i}, k_{i}}
$$

with $k_{I_{\text {ini }}}=k_{I_{\text {ini }+1}}=\ldots=k_{I_{\text {end }}}$. The remaining angles of the user $k_{I_{\text {ini }}}$ are still eligible, but they have to be pointed by other analog precoding vectors and fulfill additional interference limitations. That is, satisfying the constraints imposed due to the angular directions corresponding to the users $k_{i} \neq k_{I_{\text {ini }}}$ with positions $i$ in the sequence between $I_{\text {end }}$ and the next position assigned to user $k_{I_{\text {ini }}}$. Note also that the number of direction vectors $\boldsymbol{d}_{r}^{(n)}$ is limited by the number of RF chains, i.e., $|\mathcal{S}| \leq r \leq N_{\mathrm{RF}}$.

\section{B. Analog Precoder Design}

Once the desired precoding directions $\boldsymbol{d}_{r}^{(n)}$ are determined, we compute the corresponding analog precoding matrix $\boldsymbol{W}_{\mathrm{A}}^{0}$. To this end, let us introduce $\overline{\boldsymbol{H}}_{r}^{(n)}$ as the matrix comprising the channels corresponding to the interfering users for the $r$-th precoding direction at iteration $n$, that is,

$$
\overline{\boldsymbol{H}}_{r}^{(n)}=\left[\boldsymbol{h}_{k_{1}}, \boldsymbol{h}_{k_{2}}, \ldots, \boldsymbol{h}_{k_{C}}\right]
$$

where $k_{i} \neq k_{I_{\text {ini }}}, i=1, \ldots, C$, are the users corresponding to prior positions in the sequence $\Theta^{(n)}$, and $C \leq\left|\mathcal{S}^{(n)}\right|-1$. Hence, we propose to calculate the columns of $\boldsymbol{W}_{\mathrm{A}}^{0}$ as the solution to the following optimization problem

$$
\begin{array}{ll}
\boldsymbol{w}_{r}^{*}=\underset{\boldsymbol{w} \in \mathbb{R}^{M}}{\operatorname{argmax}} t \quad \text { s.t. } & t \geq 0, \\
& \left\|\boldsymbol{w}^{\mathrm{T}} \overline{\boldsymbol{H}}_{r}^{(n)}\right\|_{\infty} \leq \mu, \\
& \| \boldsymbol{w}_{2} \leq 1, \\
& \left|\boldsymbol{w}^{\mathrm{T}} \boldsymbol{d}_{r}^{(n)}\right|=t,
\end{array}
$$

where $\|\cdot\|_{\infty}$ is the infinity norm, and $\mu$ represents an upper bound for the interference caused to each of the users. Regardless of the group of users in $\overline{\boldsymbol{H}}_{r}^{(n)}$ and the value of $\mu$, the former problem is feasible. Thus, scheduling is fundamental to avoid trivial solutions.

Note that constraint (31) is not convex. For $\boldsymbol{w} \in \mathbb{R}^{M}$, removing the absolute value results in two constraints, namely $\boldsymbol{w}^{\mathrm{T}} \boldsymbol{d}_{r}^{(n)}=t$ and $\boldsymbol{w}^{\mathrm{T}} \operatorname{Im}\left\{\boldsymbol{d}_{r}^{(n)}\right\}=0$, which clearly degrades the achievable performance. To address this issue, we propose to follow the idea of MRT in (14) by introducing $\boldsymbol{b}_{r}^{(n)}=$ $\boldsymbol{u}_{\max }\left(\operatorname{Re}\left\{\boldsymbol{d}_{r}^{(n)}\left(\boldsymbol{d}_{r}^{(n)}\right)^{\mathrm{H}}\right\}\right)$ to obtain

$$
\begin{array}{cl}
\boldsymbol{w}_{r}^{*}=\underset{\boldsymbol{w} \in \mathbb{R}^{M}}{\operatorname{argmax}} \boldsymbol{w}^{\mathrm{T}} \boldsymbol{b}_{r}^{(n)} \quad \text { s.t. } & \left\|\boldsymbol{w}^{\mathrm{T}} \overline{\boldsymbol{H}}_{r}^{(n)}\right\|_{\infty} \leq \mu, \\
& \|\boldsymbol{w}\|_{2} \leq 1,
\end{array}
$$

which is a SOCP [27]. This kind of problems are solved by interior point methods with computational complexity $\mathcal{O}\left(M^{3}\right)$, assuming that the matrix structure is not exploited. However, the efficiency is improvable by exploting channel sparsity [27], or by using Quasy-Newtown methods [28]. In addition, the convergence rate is specially fast: $\mathcal{O}(\sqrt{M})$. Another approach to increase the efficiency is to avoid the computation of $\boldsymbol{b}_{k}$ by resorting to the asymptotically optimal approach in (15) for $\boldsymbol{d}_{r}^{(n)}$. Our numerical experiments show the suitability of this low-complexity approximation.

\section{Baseband Precoders}

Thus far only the design of the analog precoders has been considered, without taking into account the digital baseband stage. In the proposed scheme, both the scheduler and the analog precoding matrix take into account the inter-user interference. However, the scheduler only considers the effects over the users previously scheduled, and the analog precoders allow for a certain level of interference. Therefore, the baseband precoders should be helpful to remove the remaining interuser interference. 


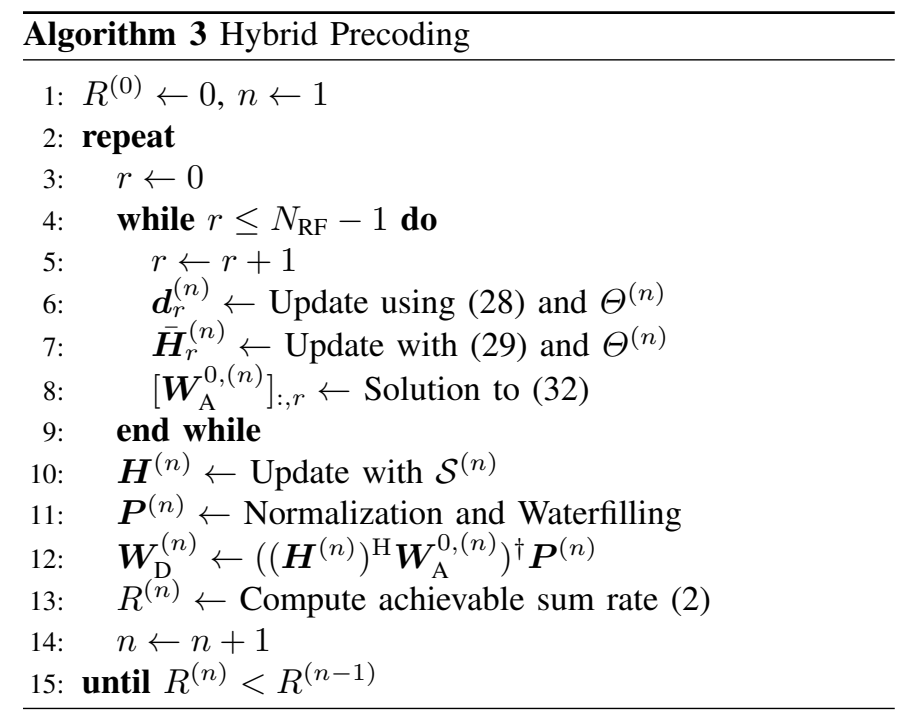

Consider that the BS serves $|\mathcal{S}| \leq N_{\mathrm{RF}}$ users and let us introduce the multiuser channel matrix $\boldsymbol{H}=\left[\boldsymbol{h}_{1}, \ldots, \boldsymbol{h}_{|\mathcal{S}|}\right] \in$ $\mathbb{C}^{M \times|\mathcal{S}|}$. Thus, the received signal can be represented as

$$
\boldsymbol{y}=\boldsymbol{H}^{\mathrm{H}} \boldsymbol{W}_{\mathrm{A}}^{0} \boldsymbol{W}_{\mathrm{D}} \boldsymbol{s}+\boldsymbol{n},
$$

with the analog and baseband precoders $\boldsymbol{W}_{\mathrm{A}}^{0} \in \mathbb{R}^{M \times N_{\mathrm{RF}}}$ and $\boldsymbol{W}_{\mathrm{D}} \in \mathbb{C}^{N_{\mathrm{RF}} \times|\mathcal{S}|}$, and the noise vector $\boldsymbol{n} \in \mathbb{C}^{|\mathcal{S}|}$. The interference is completely removed when $\boldsymbol{D}=\boldsymbol{H}^{\mathrm{H}} \boldsymbol{W}_{\mathrm{A}}^{0} \boldsymbol{W}_{\mathrm{D}}$ is a diagonal matrix. To this end we set $\boldsymbol{W}_{\mathrm{D}}$ as the $\mathrm{ZF}$ solution

$$
\boldsymbol{W}_{\mathrm{D}}=\left(\boldsymbol{H}^{\mathrm{H}} \boldsymbol{W}_{\mathrm{A}}^{0}\right)^{\dagger} \boldsymbol{P},
$$

with the diagonal matrix $\boldsymbol{P} \in \mathbb{R}_{+}^{|\mathcal{S}| \times|\mathcal{S}|}$. When the ZF constraint holds, the power allocation can be computed using waterfilling for the equivalent channel gains. To that end, the columns of $\left(\boldsymbol{H}^{\mathrm{H}} \boldsymbol{W}_{\mathrm{A}}^{0}\right)^{\dagger}$ are normalized. Next, we compute the matrix $\boldsymbol{P}$ containing the products of such normalization factors and the powers for each user, resulting from the waterfilling procedure.

Note that we carefully design the columns of the analog precoder considering the inter-user interference. In the opposite scenario where the columns of the analog precoder $\boldsymbol{W}_{\mathrm{A}}^{0}$ are independently chosen for each user, as in e.g. [14], [26], it is likely to obtain numerically unstable solutions for the precoder in (34) (see Appendix C), thus achieving low equivalent channel gains.

Algorithm 3 summarizes the proposed hybrid precoding method using the angles ordered according to Alg. 2. Note that the analog precoding matrix obtained using Alg. $3, \boldsymbol{W}_{\mathrm{A}}^{0} \in$ $\mathbb{R}^{M \times N_{\mathrm{RF}}}$, does not fulfill $\boldsymbol{W}_{\mathrm{A}}^{0} \notin \mathcal{W}$ in general. In addition, the SR has not been considered in the optimization problem. In the ensuing section, we show that identical performance is achieved for $\boldsymbol{W}_{\mathrm{A}}^{0} \in \mathcal{W}$, and propose a technique to reduce the SR.

\section{REDUCING THE SR}

In this section, we analyze the amount of power spread over the harmonic replicas of the transmitted signal. To guarantee the energy efficiency of the analog network with SPDT switches, the power losses associated to the SR must be taken into account in the design of the hybrid precoders. Observe that incorporating the total transmit power in (8) inside the problem formulation of (25) results in a difficult problem. Thus, we alternatively focus on (7), where the factor $\operatorname{sinc}\left(\pi q\left|G_{m, n}^{0}\right|\right)$ dramatically reduces for $\left|G_{m, n}^{0}\right|$ close to 1 . Accordingly, analog precoding vectors with similar absolute values for all its entries lead to high power efficiency. We thereby pose a simple but effective transformation of the objective function in (32), that is,

$$
\boldsymbol{w}^{\mathrm{T}} \boldsymbol{b}_{r}^{(n)}+\gamma\left(\min _{i}[\boldsymbol{w}]_{i}-\max _{j}[\boldsymbol{w}]_{j}\right),
$$

where $\gamma$ represents the trade-off between performance and power efficiency. This new objective function preserves convexity, and the penalty term is intended to reduce the difference between the maximum and minimum elements in $\boldsymbol{w}$. Consider the following scenarios

1) $\min _{i}[\boldsymbol{w}]_{i}<0$ and $\max _{j}[\boldsymbol{w}]_{j}<0$. Then, the penalty term is a negative number that decreases with the difference between the two negative numbers.

2) $\min _{i}[\boldsymbol{w}]_{i}>0$ and $\max _{j}[\boldsymbol{w}]_{j}>0$. Again, the difference between the two values results in the penalty term.

3) $\min _{i}[\boldsymbol{w}]_{i}<0$ and $\max _{j}[\boldsymbol{w}]_{j}>0$. If the maximum and minimum vector entries have similar absolute values the penalty term is small, and entails larger reductions in the cost function when the difference between the absolute values increases.

After solving the SOCP using (35), the analog precoding vectors are scaled with $\lambda_{1}, \ldots, \lambda_{N_{\mathrm{RF}}}$, where $\lambda_{r}=$ $\frac{1}{\max _{i} \mid\left[\boldsymbol{W}_{\mathrm{A}}^{0}\right]_{i, r},}$, therefore forcing the absolute values of the matrix entries being close to 1 . These factors do not affect the power allocated to each column of the analog precoder design, since they are readily compensated with the baseband precoders as follows $\boldsymbol{W}_{\mathrm{A}}^{0, \prime}=\boldsymbol{W}_{\mathrm{A}}^{0} \boldsymbol{\Lambda}$ and $\boldsymbol{W}_{\mathrm{D}}^{\prime}=\boldsymbol{\Lambda}^{-1} \boldsymbol{W}_{\mathrm{D}}$. This idea also applies to the obtaining of an analog precoder $\boldsymbol{W}_{\mathrm{A}}^{0, \prime}$ satisfying $\boldsymbol{W}_{\mathrm{A}}^{0, \prime} \in \mathcal{W}$ with identical performance results as the relaxed version $\boldsymbol{W}_{\mathrm{A}}^{0} \in \mathbb{R}^{M \times N_{\mathrm{RF}}}$.

\section{NUMERICAL RESULTS}

In this section, we present the results of computer experiments carried out to assess the performance of the proposed analog network with SPDT switches, scheduler, and hybrid precoding designs. We consider a setup with a BS equipped with $M=100$ antennas and $N_{\mathrm{RF}}=8 \mathrm{RF}$ chains, serving $K=16$ users. We average over 500 channel realizations according to the channel model in (4), with $L_{1}=\ldots=L_{K}=8$ random angles generated employing different distributions. Complex gains are generated according to a standard normal distribution.

In order to cancel the interference, we consider two different approaches. A first approximation is to fix the parameter in the denominator of (17) as $\beta \approx 0$ so that the scheduler approximates a zero-interference strategy. Next, the analog precoding vector is obtained by setting the interference parameter to $\mu=\infty$ in (32) to prioritize SNR maximization. In this approach, the computation of the analog precoders is very efficient since the interference cancellation is relegated to the scheduler and the first constraint of (32) vanishes. Contrarily, 


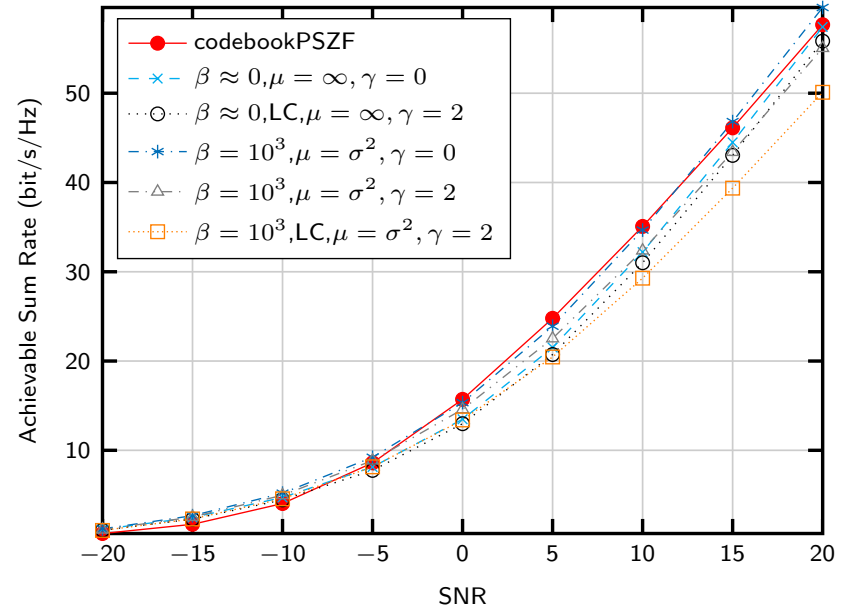

Fig. 5: Achievable sum rate vs SNR for different scheduling and precoding parameters, for uniformly distributed random angles $\vartheta_{l, k} \sim \mathcal{U}(0, \pi)$.

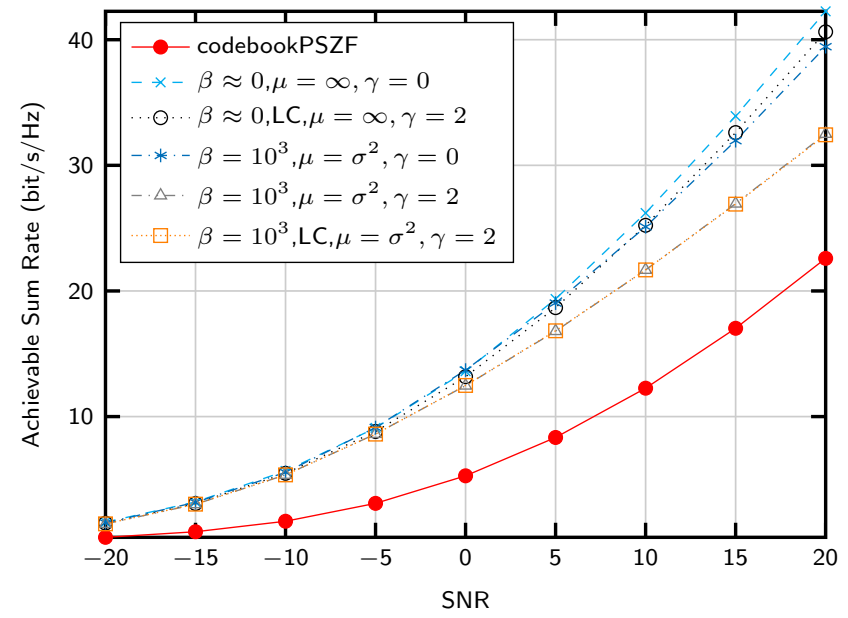

Fig. 6: Achievable sum rate vs SNR for different scheduling and precoding parameters, for clustered users $\vartheta_{l, k} \sim \mathcal{N}(\vartheta, 4)$ and $\vartheta \sim \mathcal{U}(0, \pi)$.

a second approach is to maximize the signal power using the scheduler with $\beta=10^{3}$ and bound the inter-user interference in (32) with $\mu=\sigma^{2}$. Recall that, in both configurations, the remaining interference after the multiplication times the analog precoder will be handled by the baseband precoder. In addition, when we consider the SR in (32), the parameter $\gamma$ in (35) is set as $\gamma=2$. Finally, the low complexity approach, labeled as $L C$, employs the scheduler presented in Sec. IV-D.

\section{A. Achievalbe Sum Rate}

To evaluate the throughput obtained with the aforementioned approaches, we consider the achievable sum rate as the figure of merit. As a reference, we depict the performance of analog networks with PSs (see Fig. 2(a)). In such case, we consider a scheduler that selects the users with the best channels. This simple search presents comparable computational complexity to that of the proposed scheduler. Then, we perform a codebook search for each user with the aim of

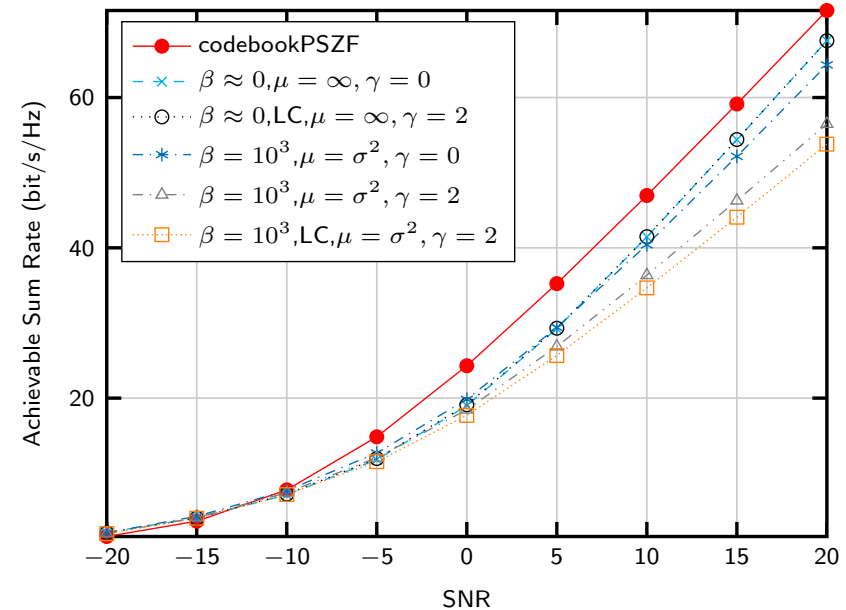

Fig. 7: Achievable sum rate vs SNR for different scheduling and precoding parameters, for clustered propagation paths for each user $\vartheta_{l, k} \sim \mathcal{N}\left(\vartheta_{k}, 4\right)$, and $\vartheta_{k} \sim \mathcal{U}(0, \pi)$.

maximizing the signal strength, followed by a ZF solution for the baseband precoders. This benchmark is referred to in the figures as codebookPSZF. The performance of this benchmark, however, is somewhat optimistic since it does not consider the signal power leakage caused by the codebook mismatch [29], nor the performance loss due to phase quantization in the PSs. Fig. 5 shows the results obtained for uniformly distributed random angles $\vartheta_{l, k} \sim \mathcal{U}(0, \pi)$. We compare the two aforementioned approximations that cancel the inter-user interference, and include the $L C$ scheduler for both of them. As shown in Fig. 5, the proposed methods throughput is similar to that of codebookPSZF, with slightly better performance in the low SNR regime. Moreover, the effect of including the $\mathrm{SR}$ in the objective function is rather limited. Indeed, the combination of the power efficient analog precoder and the $L C$ scheduler has a performance impact lower than $10 \%$ for the first approximation on the whole SNR regime, and reduces even more in the case of low and high SNR values. For the second approach, the gap between incorporating the SR and the $L C$ scheduler increases with the SNR, that is, when the inter-user interference constraints of (32) are more restrictive.

To provide a wider view of the applicability of the considered methods, we evaluate them in quite distinct scenarios. We start considering in Fig. 6 the scenario with local common scatterers at both the BS and the users analyzed in, e.g. [30], [31]. To that end, the random angles $\vartheta_{l, k}$ are generated as follows. First, we generate a uniform distributed central angle $\vartheta \sim \mathcal{U}(0, \pi)$, and the angles are then drawn according to a truncated Gaussian $\vartheta_{l, k} \sim \mathcal{N}(\vartheta, 4)$ centered at $\vartheta$. In this scenario the inter-user interference is very strong, but the proposed scheduler and analog precoding designs provide reasonable performance results. Contrarily, the achievable rate of codebookPSZF for analog networks with PSs suffers a severe degradation due to the poor equivalent channel gains obtained with this scheme (see App. C). Again, a scheduling policy focusing on the reduction of the interference shows robustness against the perturbations introduced to combat the 
SR and the application of the $L C$ version of the metric $f(\cdot)$.

The opposite setup is now evaluated by determining random angles $\vartheta_{l, k}$ as $\vartheta_{l, k} \sim \mathcal{N}\left(\vartheta_{k}, 4\right)$, for $\vartheta_{k} \sim \mathcal{U}(0, \pi)$. That is, the angles corresponding to different paths of the same user are clustered, and the inter-user interference is very small in general. Hence, an arrangement favoring the strongest angles is a good approximation in this case. Still, the proposed methods present reasonable performance. Nevertheless, the applicability of this setup to practical situations is fairly difficult.

\section{B. SR Reduction}

To evaluate the SR incurred by the TMA, we depict in Fig. 8 the total transmit power efficiency $\eta_{\mathrm{TX}} \eta_{\mathrm{OFF}}$ [see (9) and (11)] obtained with the analog network with SPDT switches, and compare with that achieved using the analog network with SPST switches [4]. To distinguish between the two methods, the markers have been removed for the curves corresponding to the analog network with SPST switches.

Thanks to the proposed objective function, we achieve efficiencies above $90 \%$. In particular, the first approximation is over $94 \% \approx-0.25 \mathrm{~dB}$. These reduced losses are in contrast to the large insertion losses caused by the use of PSs, especially when the carrier frequency $\omega_{c}$ is large like in mmWave [4], [15]. Therefore, the total transmit power employed to achieve the performance results of Fig. 5 might be much larger for the curve corresponding to the benchmark codebookPSZF, depending on many factors such as the PS technology or the carrier frequency $\omega_{c}$ [4]. For instance, using the reference values for a 4-bit PS, a 1-bit PS [4], a SPDT [32], and an adder [33], the power savings are about $2 \mathrm{~dB}$. The remarkable achievable performance, together with the superior efficiency, reveals the great interest of the proposed analog precoder design.

The power spread in the harmonics of $\omega_{c}$ when using TMAs entails a strong limitations on the applicability of these antenna arrays. In Fig. 9, we represent the efficiency of the received power $\eta_{\mathrm{RX}}$ in (10). Again, we compare with the analog network with SPST switches. The unproductive power radiated in the harmonic frequencies reduces thanks to the unitary rotations introduced by Alg. 1. This reduction is significant for the SNR regime expected at mmWave frequencies. Furthermore, combined with the SR reduction tackled with the analog precoders, some hybrid precoding designs exhibit efficiencies over $96 \%$, thus making it possible the interesting extension of this methods to wideband scenarios.

Overall, setting the scheduler parameters to attain low inter-user interference and a simpler analog precoder design, where the interference is neglected, provides very affordable computational complexity, large power efficiencies and good performance results. Moreover, these performance results show that the power divided over several angular directions can be profitably utilized employing analog networks with SPDT switches.

\section{CONCLUSIONS}

In this work we present a TMA analog network with SPDT switches to improve the OFF-state efficiency of previous TMA

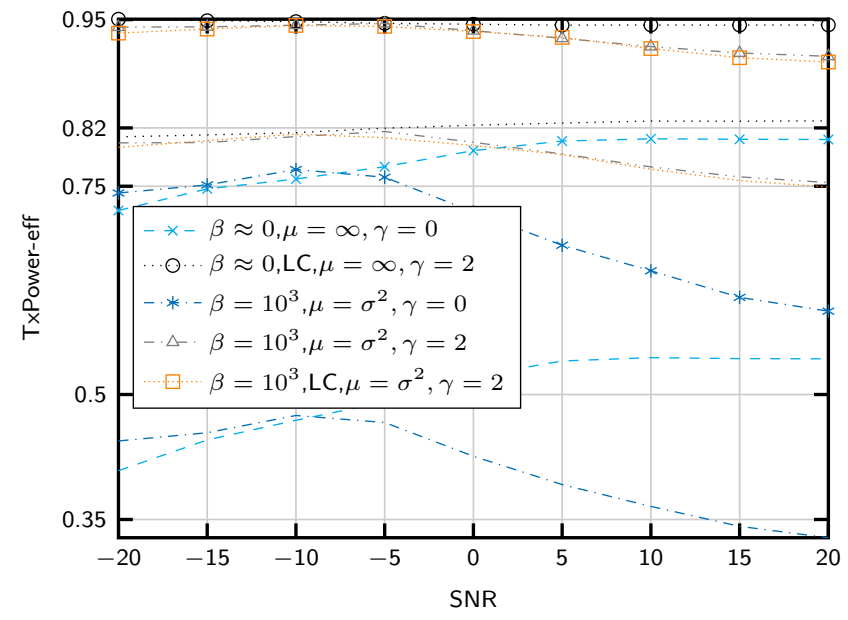

Fig. 8: Transmit power efficiency vs SNR for different scheduling and precoding parameters, for $\vartheta_{l, k} \sim \mathcal{U}(0, \pi)$.

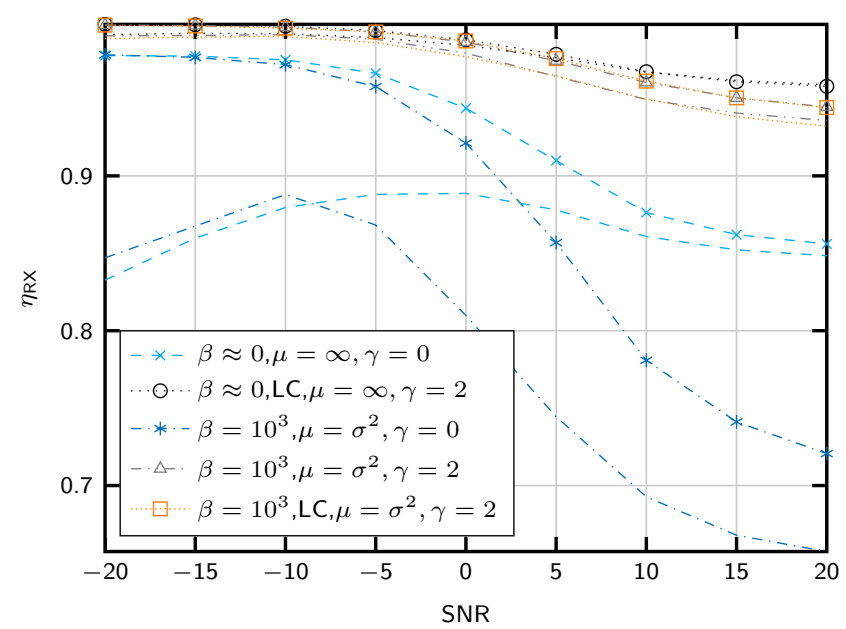

Fig. 9: Receive power efficiency vs SNR for different scheduling and precoding parameters, the posed and conventional architectures, and $\vartheta_{l, k} \sim \mathcal{U}(0, \pi)$.

hybrid digital-analog precoding solutions, without limitations in the flexibility of the analog precoder design. Moreover, we propose an angular scheduler that carefully selects the most promising angular directions of each user to avoid the inter-user interference and to maximize the received signal power. Next, the analog precoder is designed as the solution to a convex optimization problem. In addition, the SR is incorporated to this problem formulation to obtain analog precoders with very high power efficiencies, specially when compared to that of conventional analog networks with highresolution PSs.

\section{APPENDIX A ASYMPTOTICALLY OPTIMALITY}

To prove that $\boldsymbol{w}_{R}$ is an asymptotically optimal solution to (14), we first decompose the steering vector corresponding to the target angle as $\boldsymbol{a}\left(\vartheta_{t}\right)=\boldsymbol{a}_{R}\left(\vartheta_{t}\right)+\mathrm{j} \boldsymbol{a}_{I}\left(\vartheta_{t}\right)$, with $\boldsymbol{a}_{R}\left(\vartheta_{t}\right)=$ 
$\operatorname{Re}\left\{\boldsymbol{a}\left(\vartheta_{t}\right)\right\}$ and $\boldsymbol{a}_{I}\left(\vartheta_{t}\right)=\operatorname{Im}\left\{\boldsymbol{a}\left(\vartheta_{t}\right)\right\}$. Then, we get

$$
\left|\boldsymbol{w}_{R}^{\mathrm{T}}\left(\vartheta_{t}\right) \boldsymbol{a}\left(\vartheta_{t}\right)\right|^{2}=\left(\boldsymbol{w}_{R}^{\mathrm{T}}\left(\vartheta_{t}\right) \boldsymbol{a}_{R}\left(\vartheta_{t}\right)\right)^{2}+\left(\boldsymbol{w}_{R}^{\mathrm{T}}\left(\vartheta_{t}\right) \boldsymbol{a}_{I}\left(\vartheta_{t}\right)\right)^{2} .
$$

Note that the second term was neglected in the beamforming design of (15). Using $\boldsymbol{w}_{R}\left(\vartheta_{t}\right)=\frac{\boldsymbol{a}_{R}\left(\vartheta_{t}\right)}{\left\|\boldsymbol{a}_{R}\left(\vartheta_{t}\right)\right\|_{2}}$ leads to

$$
\begin{aligned}
\boldsymbol{w}_{R}^{\mathrm{T}}\left(\vartheta_{t}\right) \boldsymbol{a}_{I}\left(\vartheta_{t}\right) & =\frac{\sum_{n=0}^{M-1} \cos \left(-\pi n \cos \vartheta_{t}\right) \sin \left(-\pi n \cos \vartheta_{t}\right)}{M\left\|\boldsymbol{a}_{R}\left(\vartheta_{t}\right)\right\|_{2}} \\
& =\frac{\sum_{n=0}^{M-1} \sin \left(-2 \pi n \cos \vartheta_{t}\right)}{M\left\|\boldsymbol{a}_{R}\left(\vartheta_{t}\right)\right\|_{2}} .
\end{aligned}
$$

From the previous expression it is clear that, in the asymptotic regime,

$\lim _{M \rightarrow \infty} \boldsymbol{w}_{R}^{\mathrm{T}}\left(\vartheta_{t}\right) \boldsymbol{a}_{I}\left(\vartheta_{t}\right)=0$, thus completing the proof.

A similar reasoning applies to channel vectors $h$ with independently random real and imaginary parts $\boldsymbol{h}=\boldsymbol{h}_{R}+\mathrm{j} \boldsymbol{h}_{I}$. According to the conventional assumptions of the channel model in (4), $\boldsymbol{h}$ is a linear transformation of the vector $\boldsymbol{\alpha} \sim \mathcal{N}_{\mathbb{C}}\left(\mathbf{0}, \mathbf{I}_{M}\right)$ comprising the gains for the different paths. Therefore, $\boldsymbol{h}_{R}$ and $\boldsymbol{h}_{I}$ contain statistically independent random Gaussian entries. Then, $\boldsymbol{w}_{R}=\frac{\boldsymbol{h}_{R}}{\left\|\boldsymbol{h}_{R}\right\|_{2}}$ satisfies $\lim _{M \rightarrow \infty} \boldsymbol{w}_{R}^{\mathrm{T}} \boldsymbol{h}_{I}=0$. Thus, $\boldsymbol{w}_{R}$ is also the asymptotic MRT for channels following the model in (4).

\section{APPENDIX B}

\section{DERIVATION OF BOUNDS FOR REAL BEAMFORMERS}

Here we derive the attenuation bounds for real beamformers. Let us start by introducing the relationship

$$
\begin{aligned}
\left|\sin \left(\frac{\pi}{2} \varepsilon\right)\right| & =\mid \sin \left(\frac{\pi}{2} \cos \vartheta\right) \cos \left(\frac{\pi}{2} \cos \vartheta_{t}\right) \\
& -\cos \left(\frac{\pi}{2} \cos \vartheta\right) \sin \left(\frac{\pi}{2} \cos \vartheta_{t}\right) \mid \\
& \leq \mid-\sin \left(\frac{\pi}{2} \cos \vartheta\right) \cos \left(\frac{\pi}{2} \cos \vartheta_{t}\right) \\
& -\cos \left(\frac{\pi}{2} \cos \vartheta\right) \sin \left(\frac{\pi}{2} \cos \vartheta_{t}\right)|=| \sin \left(\frac{\pi}{2} \epsilon\right) \mid,
\end{aligned}
$$

which holds since all the factors are positive for $\vartheta_{t}, \vartheta \in\left[0, \frac{\pi}{2}\right]$. To get (24), we derive the bound

$$
\begin{aligned}
\left|\boldsymbol{w}_{s}^{\mathrm{T}}\left(\vartheta_{t}\right) \boldsymbol{a}(\vartheta)\right| & \leq \frac{\frac{1}{\left|M \sin \left(\frac{\pi}{2} \varepsilon\right)\right|}+\frac{1}{\left|M \sin \left(\frac{\pi}{2} \epsilon\right)\right|}}{2\left\|\boldsymbol{a}_{R}(\vartheta)\right\|_{2}} \\
& \leq \frac{1}{\left\|\boldsymbol{a}_{R}(\vartheta)\right\|_{2}} \frac{1}{\left|M \sin \left(\frac{\pi}{2} \varepsilon\right)\right|} .
\end{aligned}
$$

For the approach in (23), we can find the angles fulfilling the constraints of (20) for the bound $\hat{\zeta}$. Considering $\vartheta_{t} \geq \vartheta_{L}$, we equate $\hat{\zeta}$ to (23), as follows,

$$
\begin{aligned}
\hat{\zeta} & =\frac{1}{2\left\|\boldsymbol{a}_{R}\left(\vartheta_{t}\right)\right\|_{2} M}\left(\frac{\sin \left(\frac{\pi}{2} \epsilon\right)-\sin \left(\frac{\pi}{2} \varepsilon\right)}{\sin \left(\frac{\pi}{2} \varepsilon\right) \sin \left(\frac{\pi}{2} \epsilon\right)}\right) \\
& =\frac{2}{\left\|\boldsymbol{a}_{R}\left(\vartheta_{t}\right)\right\|_{2} M}\left(\frac{\sin \left(\frac{\pi}{4}(\epsilon-\varepsilon)\right) \cos \left(\frac{\pi}{4}(\epsilon+\varepsilon)\right)}{\cos \left(\frac{\pi}{2}(\varepsilon-\epsilon)\right)-\cos \left(\frac{\pi}{2}(\epsilon+\varepsilon)\right.}\right) \\
& =\frac{1}{\left\|\boldsymbol{a}_{R}\left(\vartheta_{t}\right)\right\|_{2} M}\left(\frac{\sin \left(\frac{\pi}{2} \cos \vartheta_{L}\right) \cos \left(\frac{\pi}{2} \cos \vartheta_{t}\right)}{\sin ^{2}\left(\frac{\pi}{2} \cos \vartheta_{L}\right)-\sin ^{2}\left(\frac{\pi}{2} \cos \vartheta_{t}\right)}\right) .
\end{aligned}
$$

Hence, we readily obtain the following equality

$$
a \sin ^{2}\left(\frac{\pi}{2} \cos \vartheta_{L}\right)+b \sin \left(\frac{\pi}{2} \cos \vartheta_{L}\right)+c=0,
$$

with $a=\hat{\zeta}\left\|\boldsymbol{a}_{R}\left(\vartheta_{t}\right)\right\|_{2} M, \quad b=-\cos \left(\frac{\pi}{2} \cos \vartheta_{t}\right)$, and $c=-\sin ^{2}\left(\frac{\pi}{2} \cos \vartheta_{t}\right)$. Finally, if a feasible angle exists, it is obtained as $\vartheta_{L}=\arccos \left(\frac{2}{\pi} \arcsin \left(x_{1}\right)\right)$ or $\vartheta_{L}=$ $\arccos \left(\frac{2}{\pi} \arcsin \left(x_{2}\right)\right)$, for $x_{1}$ and $x_{2}$ the solutions to (37). A similar derivation is possible to get $\vartheta_{U} \geq \vartheta_{t}$.

\section{APPENDIX C}

\section{Numerical Stability of Baseband PRecoder}

In order to design the baseband precoder $\boldsymbol{W}_{\mathrm{B}}$ in (34), we need to find the pseudo-inverse of the equivalent channel $\boldsymbol{H}^{\mathrm{H}} \boldsymbol{W}_{\mathrm{A}}$, with $\boldsymbol{W}_{\mathrm{A}} \in \mathbb{C}^{M \times N_{\mathrm{RF}}}$ and $\left|\left[\boldsymbol{W}_{\mathrm{A}}\right]_{m, n}\right|=1 \forall m, n$ for the analog network of Fig. 2a. Let us assume $N_{\mathrm{RF}}=K$ for ease of exposition. A general form of the right pseudo-inverse based on the singular value decomposition $\boldsymbol{H}^{\mathrm{H}} \boldsymbol{W}_{\mathrm{A}}=\boldsymbol{U} \boldsymbol{S} \boldsymbol{V}^{\mathrm{H}}$ is $\left(\boldsymbol{H}^{\mathrm{H}} \boldsymbol{W}_{\mathrm{A}}\right)^{\dagger}=\boldsymbol{V} \boldsymbol{S}^{\dagger} \boldsymbol{U}^{\mathrm{H}}$. Notice that, when the condition number of $\boldsymbol{H}^{\mathrm{H}} \boldsymbol{W}_{\mathrm{A}}, \frac{\max _{i}[\boldsymbol{S}]_{i, i}}{\min _{j}[\boldsymbol{S}]_{j, j}}$, is large (or infinite), a single spatial dimension is dominant in $\boldsymbol{W}_{\mathrm{B}}$. The minimum non-zero singular value is bounded by [34]

$$
\begin{aligned}
\min _{j}[\boldsymbol{S}]_{j, j} & \geq(K-1)^{\frac{K-1}{2}} \frac{\left|\operatorname{det}\left(\boldsymbol{H}^{\mathrm{H}} \boldsymbol{W}_{\mathrm{A}}\right)\right|}{\left\|\boldsymbol{H}^{\mathrm{H}} \boldsymbol{W}_{\mathrm{A}}\right\|_{\mathrm{F}}^{K-1}} \\
& =(K-1)^{\frac{K-1}{2}}|\operatorname{det}(\boldsymbol{H})| \frac{\sqrt{\operatorname{det}\left(\boldsymbol{W}_{\mathrm{A}}^{\mathrm{H}} \boldsymbol{W}_{\mathrm{A}}\right)}}{\left\|\boldsymbol{H}^{\mathrm{H}} \boldsymbol{W}_{\mathrm{A}}\right\|_{\mathrm{F}}^{K-1}} \\
& \geq(K-1)^{\frac{K-1}{2}} \frac{|\operatorname{det}(\boldsymbol{H})|}{\|\boldsymbol{H}\|_{\mathrm{F}}^{K-1}} \frac{\sqrt{\operatorname{det}\left(\boldsymbol{W}_{\mathrm{A}}^{\mathrm{H}} \boldsymbol{W}_{\mathrm{A}}\right)}}{\left\|\boldsymbol{W}_{\mathrm{A}}\right\|_{\mathrm{F}}^{K-1}} .
\end{aligned}
$$

Since $\left\|\boldsymbol{W}_{\mathrm{A}}\right\|_{\mathrm{F}}$ is fixed for fixed modulus entries in $\boldsymbol{W}_{\mathrm{A}}$, we aim at maximizing $\sqrt{\operatorname{det}\left(\boldsymbol{W}_{\mathrm{A}}^{\mathrm{H}} \boldsymbol{W}_{\mathrm{A}}\right)}$. According to Hadamard's inequality, the determinant is maximized when $\boldsymbol{W}_{\mathrm{A}}^{\mathrm{H}} \boldsymbol{W}_{\mathrm{A}}$ is diagonal

$$
\operatorname{det}\left(\boldsymbol{W}_{\mathrm{A}}^{\mathrm{H}} \boldsymbol{W}_{\mathrm{A}}\right) \leq \prod_{n=1}^{N_{\mathrm{RF}}}\left[\boldsymbol{W}_{\mathrm{A}}^{\mathrm{H}} \boldsymbol{W}_{\mathrm{A}}\right]_{n, n} .
$$

In other words, the bound of (38) is maximized when the inner products of the columns of $\boldsymbol{W}_{\mathrm{A}}$ are zero values. Let us consider that the angular directions for all the users are independently drawn from a uniform distribution in the $[0, \pi]$ interval. Using analog precoders pointing to the angular directions corresponding to the largest path gains we have $\boldsymbol{W}_{\mathrm{A}}=\left[\boldsymbol{a}\left(\vartheta_{1}\right), \ldots, \boldsymbol{a}\left(\vartheta_{K}\right)\right]$. Hence, the probability of obtaining a numerically stable $\boldsymbol{W}_{\mathrm{B}}$ can be obtained as $P\left(\left|\boldsymbol{W}_{\mathrm{A}}^{\mathrm{H}} \boldsymbol{W}_{\mathrm{A}}-\mathbf{I}_{K}\right| \leq \nu\left(\mathbf{1}_{K} \mathbf{1}_{K}^{\mathrm{T}}-\mathbf{I}_{K}\right)\right)$ for a small value of $\nu$, e.g., $\nu \ll \frac{1}{K}$. This probability, however, is difficult to calculate since the inverse function of $\left|\boldsymbol{a}^{\mathrm{H}}\left(\vartheta_{j}\right) \boldsymbol{a}\left(\vartheta_{k}\right)\right|$ does not exist.

Alternatively, we compute the probability of incorporating an additional user satisfying the desired properties. Now, if the $K-1$ columns of $\boldsymbol{W}_{\mathrm{A}}$ present small inner products, we have

$$
\begin{aligned}
P\left(\left|\boldsymbol{W}_{\mathrm{A}}^{\mathrm{H}}\left(\vartheta_{K}\right) \boldsymbol{a}\left(\vartheta_{k}\right)\right|\right. & \left.\leq \nu \mathbf{1}_{K-1}\right) \\
& =\prod_{k=1}^{K-1} P\left(\left|\boldsymbol{a}^{\mathrm{H}}\left(\vartheta_{K}\right) \boldsymbol{a}\left(\vartheta_{k}\right)\right| \leq \nu\right) .
\end{aligned}
$$

Resorting to (21), we obtain for each user $k \in\{1, \ldots, K-1\}$

$$
\begin{aligned}
P\left(\left|\boldsymbol{a}^{\mathrm{H}}\left(\vartheta_{K}\right) \boldsymbol{a}\left(\vartheta_{k}\right)\right| \leq \nu\right) & =P\left(\vartheta_{K} \leq \vartheta_{L}\right)+P\left(\vartheta_{K} \geq \vartheta_{U}\right) \\
& =1-\frac{2}{\pi}\left(\vartheta_{U}-\vartheta_{L}\right),
\end{aligned}
$$


with $\vartheta_{L}=\arccos \left(\cos \vartheta_{k}+\frac{2}{\pi} \arcsin \left(\frac{1}{\nu M}\right)\right)$ and $\vartheta_{U}=$ $\arccos \left(\cos \vartheta_{k}-\frac{2}{\pi} \arcsin \left(\frac{1}{\nu M}\right)\right)$. As a simple and illustrative example, setting $M=100, K=10, \nu=0.05$, and a very optimistic theoretical scenario with $\vartheta_{1}=\ldots=\vartheta_{K-1}=\frac{\pi}{2}$, gives a probability smaller than 0.5 for (39).

\section{REFERENCES}

[1] F. Rusek, D. Persson, B. K. Lau, E. G. Larsson, T. L. Marzetta, O. Edfors, and F. Tufvesson, "Scaling Up MIMO: Opportunities and Challenges with Very Large Arrays," IEEE Signal Processing Magazine, vol. 30, no. 1, pp. 40-60, January 2013.

[2] T. S. Rappaport, S. Sun, R. Mayzus, H. Zhao, Y. Azar, K. Wang, G. N. Wong, J. K. Schulz, M. Samimi, and F. Gutierrez, "Millimeter Wave Mobile Communications for 5G Cellular: It Will Work!" IEEE Access, vol. 1, pp. 335-349, 2013.

[3] O. E. Ayach, S. Rajagopal, S. Abu-Surra, Z. Pi, and R. W. Heath, "Spatially Sparse Precoding in Millimeter Wave MIMO Systems," IEEE Transactions on Wireless Communications, vol. 13, no. 3, pp. 14991513, March 2014.

[4] J. P. González-Coma, R. Maneiro-Catoira, and L. Castedo, "Hybrid Precoding With Time-Modulated Arrays for Mmwave MIMO Systems," IEEE Access, vol. 6, pp. 59422-59437, 2018.

[5] J. Bregains, J. Fondevila-Gomez, G. Franceschetti, and F. Ares, "Signal radiation and power losses of time-modulated arrays," IEEE Transactions on Antennas and Propagation, vol. 56, no. 6, pp. 1799-1804, 2008.

[6] Q. Zhu, S. Yang, R. Yao, and Z. Nie, "Gain improvement in timemodulated linear arrays using SPDT switches," IEEE Antennas and Wireless Propagation Letters, vol. 11, pp. 994-997, 2012.

[7] J. Chen, X. Liang, C. He, H. Fan, W. Zhu, R. Jin, and J. Geng, "Efficiency improvement of time modulated array with reconfigurable power divider/combiner," IEEE Transactions on Antennas and Propagation, vol. 65, no. 8, pp. 4027-4037, Aug 2017.

[8] C. He, X. Liang, B. Zhou, J. Geng, and R. Jin, "Space-division multiple access based on time-modulated array," IEEE Antennas and Wireless Propagation Letters, vol. 14, pp. 610-613, 2015.

[9] M. H. Mazaheri, M. Fakharzadeh, and M. Akbari, "Efficiency Enhancement of Time-Modulated Arrays With Optimized Switching Sequences," IEEE Transactions on Antennas and Propagation, vol. 66, no. 7, pp. 3411-3420, July 2018.

[10] X. Sun, X. Gao, G. Y. Li, and W. Han, "Agglomerative user clustering and downlink group scheduling for FDD massive MIMO systems," in Proc. IEEE International Conference on Communications (ICC), May 2017, pp. 1-6.

[11] B. Zhang, L. J. Cimini, and L. J. Greenstein, "Efficient Eigenspace Training and Precoding for FDD Massive MIMO Systems," in Proc. IEEE Global Communications Conference (GLOBECOM), Dec 2017, pp. $1-6$.

[12] J. P. González-Coma, W. Utschick, and L. Castedo, "Hybrid LISA for Wideband Multiuser Millimeter-Wave Communication Systems Under Beam Squint," IEEE Transactions on Wireless Communications, vol. 18, no. 2, pp. 1277-1288, February 2019.

[13] R. A. Stirling-Gallacher and M. S. Rahman, "Multi-user MIMO strategies for a millimeter wave communication system using hybrid beam-forming," in International Conference on Communications, (ICC), 2015, pp. 2437-2443. [Online]. Available: https://doi.org/10.1109/ICC. 2015.7248690

[14] A. Alkhateeb, G. Leus, and R. W. Heath, "Limited Feedback Hybrid Precoding for Multi-User Millimeter Wave Systems," IEEE Transactions on Wireless Communications, vol. 14, no. 11, pp. 6481-6494, November 2015.

[15] R. Méndez-Rial, C. Rusu, N. González-Prelcic, A. Alkhateeb, and R. W. Heath, "Hybrid MIMO Architectures for Millimeter Wave Communications: Phase Shifters or Switches?" IEEE Access, vol. 4, pp. 247-267, 2016.

[16] C. Rusu, R. Méndez-Rial, N. González-Prelcic, and R. W. Heath, "Low Complexity Hybrid Precoding Strategies for Millimeter Wave MIMO Communication Systems," IEEE Transactions on Wireless Communications, vol. 15, no. 12, pp. 8380-8393, 2016.

[17] A. A. M. Saleh and R. Valenzuela, "A Statistical Model for Indoor Multipath Propagation," IEEE Journal on Selected Areas in Communications, vol. 5, no. 2, pp. 128-137, February 1987.

[18] L. Poli, P. Rocca, L. Manica, and A. Massa, "Pattern synthesis in time-modulated linear arrays through pulse shifting," IET Microwaves, Antennas Propagation, vol. 4, no. 9, pp. 1157-1164, September 2010.
[19] C. Guthy, W. Utschick, and G. Dietl, "Low-complexity linear zeroforcing for the mimo broadcast channel," IEEE Journal of Selected Topics in Signal Processing, vol. 3, no. 6, pp. 1106-1117, December 2009.

[20] A. Adhikary, J. Nam, J. Y. Ahn, and G. Caire, "Joint Spatial Division and Multiplexing: The Large-Scale Array Regime," IEEE Transactions on Information Theory, vol. 59, no. 10, pp. 6441-6463, October 2013.

[21] Q. Spencer, A. Swindlehurst, and M. Haardt, "Zero-forcing methods for downlink spatial multiplexing in multiuser MIMO channels," IEEE Transactions on Signal Processing, vol. 52, no. 2, pp. 461-471, February 2004.

[22] J. A. Tropp, A. C. Gilbert, and M. J. Strauss, "Algorithms for simultaneous sparse approximation. Part I: Greedy pursuit," Signal Processing, vol. 86, no. 3, pp. 572 - 588, 2006, sparse Approximations in Signal and Image Processing. [Online]. Available: http://www.sciencedirect.com/science/article/pii/S0165168405002227

[23] J. A. Tropp and A. C. Gilbert, "Signal Recovery From Random Measurements Via Orthogonal Matching Pursuit," IEEE Transactions on Information Theory, vol. 53, no. 12, pp. 4655-4666, December 2007.

[24] M. Schubert and H. Boche, "Solution of the Multiuser Downlink Beamforming Problem with Individual SINR Constraints," IEEE Transactions on Vehicular Technology, vol. 53, no. 1, pp. 18-28, January 2004.

[25] D. Tse, Fundamentals of wireless communication. Cambridge: Cambridge University Press, 2005.

[26] S. He, J. Wang, Y. Huang, B. Ottersten, and W. Hong, "CodebookBased Hybrid Precoding for Millimeter Wave Multiuser Systems," IEEE Transactions on Signal Processing, vol. 65, no. 20, pp. 5289-5304, October 2017.

[27] M. S. Lobo, L. Vandenberghe, S. Boyd, and H. Lebret, "Applications of second-order cone programming," Linear algebra and its applications, vol. 284, no. 1-3, pp. 193-228, 1998.

[28] J. Gondzio and F. N. C. Sobral, "Quasi-newton approaches to interior point methods for quadratic problems," School of Mathematics, University of Edinburg, Tech. Rep. ERGO 18-015, June 2018. [Online]. Available: http://www.optimization-online.org/DB_FILE/2018/06/6677. pdf

[29] Y. Chi, L. L. Scharf, A. Pezeshki, and A. R. Calderbank, "Sensitivity to basis mismatch in compressed sensing," IEEE Transactions on Signal Processing, vol. 59, no. 5, pp. 2182-2195, May 2011.

[30] J. Shen, J. Zhang, E. Alsusa, and K. B. Letaief, "Compressed CSI Acquisition in FDD Massive MIMO: How Much Training is Needed?" IEEE Transactions on Wireless Communications, vol. 15, no. 6, pp. 4145-4156, June 2016.

[31] X. Cheng, J. Sun, and S. Li, "Channel Estimation for FDD Multi-User Massive MIMO: A Variational Bayesian Inference-Based Approach," IEEE Transactions on Wireless Communications, vol. 16, no. 11, pp. 7590-7602, November 2017.

[32] "Qorvo," http://www.qorvo.com, references: RFSW6024, RF1630, RFSW1012. Accessed: 2019-05-02.

[33] A. Natarajan, S. K. Reynolds, M. Tsai, S. T. Nicolson, J. C. Zhan, D. G. Kam, D. Liu, Y. O. Huang, A. Valdes-Garcia, and B. A. Floyd, "A FullyIntegrated 16-Element Phased-Array Receiver in SiGe BiCMOS for 60GHz Communications," IEEE Journal of Solid-State Circuits, vol. 46 , no. 5, pp. 1059-1075, May 2011.

[34] G. Piazza and T. Politi, "An upper bound for the condition number of a matrix in spectral norm," Journal of Computational and Applied Mathematics, vol. 143, no. 1, pp. 141 - 144, 2002. [Online]. Available: http://www.sciencedirect.com/science/article/pii/S0377042702003965 